\title{
Special Vinberg cones and the entropy of BPS extremal black holes
}

\author{
Dmitri V. Alekseevsky, ${ }^{a, b}$ Alessio Marrani ${ }^{c}$ and Andrea Spiro ${ }^{d}$ \\ ${ }^{a}$ Institute for Information Transmission Problems, \\ B. Karetnuj per. 19, Moscow 127051, Russia \\ ${ }^{b}$ University of Hradec Králové, Faculty of Science, \\ Rokitanského 62, Hradec Králové 500 03, Czech Republic \\ ${ }^{c}$ Centro Studi e Ricerche Enrico Fermi, \\ via Panisperna 89A, Roma I-00184, Italy \\ ${ }^{d}$ Scuola di Scienze e Tecnologie, Università di Camerino, \\ Via Madonna delle Carceri, Camerino I-62032, Macerata, Italy \\ E-mail: dalekseevsky@iitp.ru, jazzphyzz@gmail.com, \\ andrea.spiro@unicam.it
}

ABSTRACT: We consider the static, spherically symmetric and asymptotically flat BPS extremal black holes in ungauged $N=2 D=4$ supergravity theories, in which the scalar manifold of the vector multiplets is homogeneous. By a result of Shmakova on the BPS attractor equations, the entropy of this kind of black holes can be expressed only in terms of their electric and magnetic charges, provided that the inverse of a certain quadratic map (uniquely determined by the prepotential of the theory) is given. This inverse was previously known just for the cases in which the scalar manifold of the theory is a homogeneous symmetric space. In this paper we use Vinberg's theory of homogeneous cones to determine an explicit expression for such an inverse, under the assumption that the scalar manifold is homogeneous, but not necessarily symmetric. As immediate consequence, we get a formula for the entropy of BPS black holes that holds in any model of $N=2$ supergravity with homogeneous scalar manifold.

KEywords: Black Holes, Supergravity Models, Classical Theories of Gravity, Differential and Algebraic Geometry

ArXiv EPrint: 2107.06797 


\section{Contents}

1 Introduction 2

2 Geometry of special Vinberg cones and their duals 5

2.1 Special $T$-algebras and their standard matrix representations 5

2.1.1 Special $T$-algebras 5

2.1.2 The standard matrix representation of a special $T$ algebra $\quad 7$

2.2 Special Vinberg cones and their dual cones 9

2.2.1 The linear actions of $G$ and $G^{*}$ on the space of Hermitian matrices $\mathcal{H} \quad 9$

2.2.2 The linear representation of $G$ on the dual space $\mathcal{H}^{\prime}=\operatorname{Hom}(\mathcal{H}, \mathbb{R}) \quad 9$

$\begin{array}{ll}2.2 .3 & \text { The special Vinberg cones and their dual and adjoint cones }\end{array}$

2.3 Group coordinates, adapted orthogonal coordinates and de Wit and

$\begin{array}{ll}\text { Van Proeyen coordinates } & 10\end{array}$

2.4 The invariant and dual invariant cubic polynomials 12

2.5 Quadratic maps associated with invariant cubic polynomials and their inverses 15

3 Projective-special Kähler manifolds and very special cones 17

3.1 Conical scalar manifolds, projective scalar manifolds and special Vinberg cones 17

3.2 Description of projective scalar manifolds in terms of conical holomorphic $\begin{array}{ll}\text { coordinates and prepotentials } & 18\end{array}$

$\begin{array}{lll}3.3 & \text { The supergravity r-map } & 19\end{array}$

4 BPS black holes in $\mathcal{N}=24 D$ supergravity $\quad 20$

4.1 Scalar fields, vector fields and central charges in an $\mathcal{N}=24 D$ supergravity $\begin{array}{ll}\text { theory } & 20\end{array}$

4.2 Static and spherically symmetric black holes and their electro-magnetic charges 21

4.3 BPS black holes, their entropy and the "inverse relation" map 22

5 Recovering the central charge and the scalar fields from the electric and magnetic charges of a BPS black hole

5.1 The BPS relations as a map 24

5.2 The maps $\left.\mathfrak{f}\right|_{\mathcal{C}_{ \pm}}$take values into $\left\{\mathfrak{p}^{0}=0\right\}$ and are globally invertible 25

5.3 The maps $\left.\mathfrak{f}\right|_{\mathcal{A}_{ \pm}}$take values into $\left\{\mathfrak{p}^{0} \neq 0\right\}$ and are locally invertible 26

5.4 The entropy of BPS black holes in case of homogeneous scalar manifolds $\quad 30$ 


\section{Introduction}

The first purpose of this paper is to determine an explicit formula which gives the Bekenstein-Hawking entropy of a static, spherically symmetric and asymptotically flat BPS extremal black hole in terms of its electric and magnetic charges in ungauged $N=2 D=4$ supergravity theory, under the assumption that the scalar manifold of the vector multiplets is homogeneous. As a secondary goal, we want to offer a gentle introduction to Vinberg's theory of homogeneous cones associated with irreducible invariant cubic polynomials and to illustrate how this purely mathematical theory can be combined with some fundamental theoretical physics results, such as Bekenstein-Hawking entropy-area formula or Ferrara, Kallosh and Strominger's BPS algebraic attractor equations, to establish new non-trivial results on black holes.

The paper starts with a discussion of the invariant real cubic polynomials $d(y)=$ $d_{a b c} y^{a} y^{b} y^{c},\left(y^{a}\right) \in \mathbb{R}^{n}$, which are associated to the holomorphic prepotentials

$$
F(X)=\frac{d_{a b c} X^{a} X^{b} X^{c}}{X^{0}}, \quad\left(X^{I}\right)=\left(X^{0}, X^{a}\right) \in \mathbb{C}^{n+1},
$$

that determine homogeneous scalar manifolds of the vector multiplets of ungauged $N=2$ $D=4$ supergravity. By known results on prepotentials and associated scalar manifolds $([2,11,12,18])$, any irreducible cubic polynomial of this kind corresponds to a rank 3 homogeneous convex cone $\mathcal{V} \subset \mathbb{R}^{n}$ of dimension $n$. This is in turn representable as the cone of positive Hermitian matrices in an appropriate space of $3 \times 3$ matrices, with vector and spinor valued entries, given by a special Vinberg $T$-algebra (see $[3,40]$ and section $2.2-$ 2.3). Aiming to a presentation that might be accessible to any reader with no previous knowledge of Vinberg's theory, the first section starts with a self-contained exposition of the main definitions and properties of special Vinberg $T$-algebras, of the corresponding cones of positive Hermitian matrices and of the invariant cubic polynomials which are important objects associated with these cones. We also introduce the notions of dual cones and associated dual invariant cubic polynomials. These new objects are later used to determine the explicit general expressions for the inverses to the quadratic maps, that appear in the explicit entropy formula for BPS extremal black holes established in the third part.

The second and third parts of the paper provide a short review of special Kähler geometry and of the BPS extremal black holes in ungauged Maxwell-Einstein $N=2$ supergravity with prepotentials of the form (1.1). The presentation is structured for readers who are not familiar with supergravity. Here is a short outline. Consider the class of metrics on the 4-dimensional space-time $M$ of the form [31, 34],

$$
d s^{2}=-e^{2 U(r)} d t^{2}+e^{-2 U(r)}\left(d r^{2}+r^{2}\left(d \theta^{2}+\sin ^{2} \theta d \varphi^{2}\right)\right) .
$$

They are solutions to the Euler-Lagrange equations of the bosonic sector of the aforementioned supergravity and describe static, spherically symmetric, asymptotically flat, dyonic extremal black holes with unique event horizon at $r=0$ (as implied by extremality). The magnetic and electric charges of any such black hole are the fluxes of the electromagnetic 
fields $\mathbb{F}^{I}=\mathbb{F}_{\mu \nu}^{I} d x^{\mu} \wedge d x^{\nu}$ and their duals $\mathbb{G}_{J}=\star \frac{1}{2} \frac{\delta \mathcal{L}}{\delta \mathbb{F}^{J}}$ with respect to the Lagrangian $\mathcal{L}$ of the theory,

$$
p^{I}:=\frac{1}{4 \pi} \int_{S_{\infty}^{2}} \mathbb{F}^{I}, \quad q_{J}:=\frac{1}{4 \pi} \int_{S_{\infty}^{2}} \mathbb{G}_{J} .
$$

Due to its rotational invariance and time independence, the dynamics of the scalar and electromagnetic fields of this kind of black holes can be described by means of a reduced 1-dimensional Lagrangian, characterised by the effective black hole potential [19]

$$
V_{\mathrm{BH}}\left(p^{I}, q_{J}, z, \bar{z}\right):=|Z|^{2}+g^{a \bar{b}} Z_{a} \overline{Z_{b}} \quad \text { with } \quad Z_{a}:=\frac{\partial Z}{\partial z^{a}}+\frac{1}{2} \frac{\partial \mathcal{K}}{\partial z^{a}} Z,
$$

where (i) $z=\left(z^{a}\right)$ is the map that represents the scalar fields and takes values in the scalar manifold $\mathcal{S} \subset \mathbb{C}^{n}$; (ii) $g=\left(g_{a \bar{b}}\right)$ is the Kähler metric of the scalar manifold $\mathcal{S}$; (iii) $\mathcal{K}$ is the Kähler potential of $g$, (iv) $Z=Z\left(p^{I}, q_{J}, z, \bar{z}\right)$ is the $N=2$ central charge, which is a function of the magnetic and electric charges $\left(p^{I}, q_{J}\right)$ and the scalar fields $z=\left(z^{a}\right)$. We recall that, for any extremal black hole (1.2), the central charge function $Z=Z\left(p^{I}, q_{J}, z, \bar{z}\right)$ satisfies the identity

$$
\left(\begin{array}{c}
p^{I} \\
q_{J}
\end{array}\right)=-2 e^{\frac{\mathcal{K}}{2}} \operatorname{Im}\left(\bar{Z}\left(\begin{array}{c}
X^{I} \\
F_{J}
\end{array}\right)+g^{a \bar{b}} Z_{a} \overline{\left(\frac{\partial}{\partial z^{b}}+\frac{\partial \mathcal{K}}{\partial z^{b}}\right)\left(\begin{array}{c}
X^{I} \\
F_{J}
\end{array}\right)}\right),
$$

where $\left(X^{I}\right)=\left(\begin{array}{c}X^{0} \\ X^{a}\end{array}\right)=X^{0}\left(\begin{array}{c}1 \\ z^{a}\end{array}\right) \in \mathbb{C}^{n+1}$ with $z^{a}=\frac{X^{a}}{X^{0}}$, and $F_{J}:=\left.\frac{\partial F}{\partial X_{J}}\right|_{\left(X^{I}\right)}$.

For given charges $p^{I}, q_{J}$ in an appropriate set, it is known that the values of the scalar fields $z$ at $r=0$ are stable critical points for $V_{\mathrm{BH}}$. By the famous Attractor Mechanism [19, 22-24, 39], such criticality condition determines a (locally invertible) relation between the horizon values of the scalar fields and the magnetic and electric charges of the black hole.

Let us now focus on the black holes (1.2) which are in addition BPS. For them the following two facts hold: (a) the central charge $Z_{o}=\left.Z\right|_{r=0}$ at the horizon verifies the relation $\left|Z_{o}\right|^{2}=\frac{A_{H}}{4 \pi}$, where $A_{H}$ denotes the area of the event horizon surface; (b) all covariant derivatives $Z_{a}:=\frac{\partial Z}{\partial z^{a}}+\frac{1}{2} \frac{\partial \mathcal{K}}{\partial z^{a}} Z$ are identically zero at $r=0$ and the effective potential at the horizon reduces to $\left.V_{\mathrm{BH}}\right|_{r=0}=\left|Z_{o}\right|^{2}$. In particular, for such black holes the identities (1.5) imply the following purely algebraic relations between $Z_{o}$, the magnetic and electric charges and the values $X_{o}=\left(X_{o}^{I}\right)=X_{o}^{0}\left(\begin{array}{c}1 \\ z_{o}^{a}\end{array}\right)$, associated with the scalar fields $z_{o}:=\left.z\right|_{r=0}$ :

$$
\left(\begin{array}{l}
p^{I} \\
q_{J}
\end{array}\right)=-2 e^{\frac{\mathcal{K}\left(z_{o}\right)}{2}} \operatorname{Im}\left(\overline{Z_{o}}\left(\begin{array}{c}
X_{o}^{I} \\
F_{o J}
\end{array}\right)\right) .
$$

This is a system of equations that relates the set of the $2 n+2$ real numbers $p^{I}, q_{J}$ and the set of the $n+1$ complex numbers $\left(Z_{o}, z_{o}^{1}, \ldots, z_{o}^{n}\right)$. Such a system is always locally solvable in terms of the second set [38].

On the other hand, by the Bekenstein-Hawking entropy-area formula [7, 28], the above property (a) of the BPS metrics (1.2) implies that the entropy of any such black hole is related with the central charge $Z_{o}$ at the horizon by [19]

$$
S=\frac{A_{H}}{4}=\pi\left|Z_{o}\right|^{2}
$$


Hence any (local) inversion of the algebraic relation (1.6) (thus giving $Z_{o}$ as a function of $p^{I}$ and $q_{J}$ ) provides a formula for the black hole entropy $S$ in terms of its magnetic and electric charges. The fourth part of our paper is devoted to the solution of such inversion problem.

More precisely, in our fourth section, we analyse in detail the correspondence $\left(z_{o}^{a}, Z_{o}\right) \mapsto$ $\left(p^{I}, q_{J}\right)$ determined by (1.6), which we name BPS map. After a brief discussion about the invertibility of such a map in the (simpler) case with $p^{0}=0$, we tackle the situations with $p^{0} \neq 0$. For them, we show that the BPS map is always a local diffeomorphism. This is obtained using Shmakova's formulas in [38] (which we newly derive in detail by means of a different line of arguments) that reduce the inversion problem for the BPS map to the (somehow simpler) inversion problem of the quadratic map

$$
h_{d}: \mathbb{R}^{n} \longrightarrow \mathbb{R}^{n^{\prime}}=\operatorname{Hom}\left(\mathbb{R}^{n}, \mathbb{R}\right), \quad h_{d}(y):=\left(d_{a b c} y^{b} y^{c}\right) .
$$

From these formulas, the BPS map is proved to be a local diffeomorphism by checking that $h_{d}$ has a non-vanishing Jacobian and thus, due to the Inverse Function Theorem, it is locally invertible with smooth inverse. In the cases in which $d(y)$ is an irreducible polynomial and is associated with homogeneous scalar manifolds, a globally defined inverse for $h_{d}: \mathbb{R}^{n} \rightarrow \mathbb{R}^{n *}$ has been explicitly determined in the first part of this paper. Therefore, the results of our first part immediately yield to explicit formulas for global inverses to the BPS map, one per each of the two connected regions corresponding to the condition $p^{0} \neq 0$. These two inverse maps give expressions for the horizon values $Z_{o}, z_{o}=\left(z_{o}^{a}\right)$ in terms of the electric and magnetic charges only. By (1.7), they also give an expression for the entropy of the black hole.

At the best of our knowledge, explicit expressions for the inverse map $h_{d}^{-1}$ and, consequently, for the entropy $S$ of the above BPS black holes, was so far known only when $d(y)=d_{a b c} y^{a} y^{b} y^{c}$ and the corresponding prepotential determine a homogeneous symmetric scalar manifold $\mathcal{S}$. Our results can be thus considered as a completion of such previous results, providing a solution to the above BPS black hole entropy problem for all cases in which $\mathcal{S}$ is homogeneous, regardless whether it is symmetric or non-symmetric.

The expression for the entropy $S$ we obtain for the considered cases is

$$
\begin{gathered}
S=\pi \sqrt{I_{4}} \text {, where } I_{4}=I_{4}\left(p^{0}, p^{a}, q_{0}, q_{b}\right) \text { is the } 4^{\text {th }} \text { order polynomial } \\
I_{4}\left(p^{0}, p^{a}, q_{0}, q_{b}\right)=-\left(p^{0} q_{0}+p^{a} q_{a}\right)^{2}+4 q_{0} d_{a b c} p^{a} p^{b} p^{c}-\frac{4}{27} p^{0} d^{a b c} q_{a} q_{b} q_{c}+\frac{4}{3} d_{a b c} d^{a d e} p^{b} p^{c} q_{d} q_{e} .
\end{gathered}
$$

Here $d^{*}(w)=d^{a b c} w_{a} w_{b} w_{c}$ is the dual invariant cubic polynomial associated with $d(y)$, which we define in definition 2.7 and we explicitly determine in theorem 2.8. As we have already pointed out, formula (1.8) was previously known just in the cases in which $(\mathcal{S}=G / K, g)$ is a homogeneous symmetric Hermitian space. In all such cases it was observed by the second author in [32] that, up to a factor, the natural extension of the polynomial $I_{4}$ to $\mathbb{C}^{2 n+2}$ coincides with the unique generator of the ring of the relative invariants of the standard representation of $\mathbb{C}^{*} \times \rho(G)^{\mathbb{C}}=\left(\mathbb{R}_{+} \times \rho(G)\right)^{\mathbb{C}}$ on $\mathbb{C}^{2 n+2}$, where $\rho: G \hookrightarrow \operatorname{Sp}_{2 n+2}(\mathbb{R})$ is an 
appropriate embedding of $G$ into $\operatorname{Sp}_{2 n+2}(\mathbb{R})$. This remarkable property is a consequence of the following three facts: (a) the equations of motion of the supergravity that we consider are invariant under the group $G_{\text {e.m. }}$ of the electric-magnetic dualities (also called generalised duality transformations or $U$-dualities in supergravity literature); (b) $G_{\text {e.m. }}$ is a subgroup of $\operatorname{Sp}_{2 n+2}(\mathbb{R})$ acting in a standard way on the space $\mathbb{R}^{2 n+2}$ of the magnetic and electric charges of the above BPS black holes and admitting a natural isomorphism $\sigma=\rho^{-1}$ : $G_{\text {e.m. }} \rightarrow G=\operatorname{Iso}^{o}(\mathcal{S}, g)$ with the identity component $G=\operatorname{Iso}^{o}(\mathcal{S}, g)$ of the isometry group Iso $(\mathcal{S}, g)$; (c) the standard representation of $\mathbb{C}^{*} \times\left(G_{\text {e.m. }}\right)^{\mathbb{C}}$ on $\mathbb{C}^{2 n+2}$ has an open orbit. Note that (c) is precisely the property that implies that $I_{4}$ is the unique generator (up to a scaling) for the ring of the relative invariants.

Since (a) and (b) are true whenever $(\mathcal{S}=G / K, g)$ is a homogeneous (not necessarily symmetric) manifold, we immediately have that in all these cases $I_{4}$ is a relative invariant for the action of $\left(\mathbb{R}_{+} \times \rho(G)\right)^{\mathbb{C}}, G^{\mathbb{C}} \subset \mathrm{Sp}_{2 n+2}(\mathbb{C})$, on $\mathbb{C}^{2 n+2}$. But we claim that also (c) is true for any homogeneous scalar manifold. In fact, from the explicit expression of the BPS map and recalling the explicit form of the linear action of $G$ on $\mathbb{C}^{n+1}$ (which projects onto the scalar manifold $\mathcal{S}$ under projectivisation), one can directly check that the isotropy subgroup of the representation $\rho(G)=G_{\text {e.m. }}$ on $\mathbb{R}^{2 n+2}$ has one dimension less than the isotropy $H$ of the scalar manifold $\mathcal{S}=G / H$. Thus the regular orbits $\rho(G) \cdot\left(p^{I}, q_{J}\right) \subset \mathbb{R}^{2 n+2}$

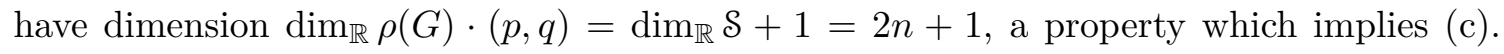
Combining these three simple observations, we may conclude that (up to a rescaling) $I_{4}$ is the unique generator of the relative invariants of $\left(\mathbb{R}_{+} \times \rho(G)\right)^{\mathbb{C}}$ for all cases in which $\mathcal{S}$ is homogeneous. Details on this and other aspects of the quartic polynomial $I_{4}$ are left to a future work.

\section{Acknowledgments}

The authors are sincerely grateful to the Referee for her/his constructive criticism, who helped to consistently improve the presentation.

\section{Geometry of special Vinberg cones and their duals}

\subsection{Special $T$-algebras and their standard matrix representations}

\subsubsection{Special $T$-algebras}

Let $\left(V, g_{V}\right)$ be a Euclidean vector space with associated Clifford algebra $\mathcal{C} \ell(V)$, constructed according to the Clifford relation $v \cdot w+w \cdot v=-2 g_{V}(v, w)$. Let also $S=S_{0}+S_{1}$ be a $\mathbb{Z}_{2^{-}}$ graded $\mathcal{C} \ell(V)$ module equipped with a Euclidean scalar product $g_{S}$ satisfying the following two conditions:

(1) $g_{S}\left(S_{0}, S_{1}\right)=0$

(2) each Clifford multiplication $\mu(v, \cdot): S \rightarrow S, v \in V$, is $g_{S}$-skew symmetric.

In the terminology of $[1,2]$, a scalar product satisfying these conditions is called symmetric admissible scalar product of type $\tau=-1$.

We call the space $\left(S, g_{S}\right)$ a metric $\mathcal{C} \ell(V)$-module. 
In the following, to simplify notation, we will denote both the metric $g_{V}+g_{S}$ of $V+S$ and the corresponding induced metric on $V^{\prime}+S^{\prime}=\operatorname{Hom}(V+S, \mathbb{R})$ by $\langle\cdot, \cdot\rangle$. We will also denote by $(\cdot)^{b}: V+S \rightarrow V^{\prime}+S^{\prime}$ the associated isomorphism

$$
v+s \mapsto v^{b}+s^{b}=\langle v+s, \cdot\rangle,
$$

by $(\cdot)^{\sharp}: V^{\prime}+S^{\prime} \rightarrow V+S$ the inverse map of $(\cdot)^{b}$ and by $\langle\cdot, \cdot\rangle_{V^{\prime}+S^{\prime}}=g_{V^{\prime}}+g_{S^{\prime}}$ the scalar product on $V^{\prime}+S^{\prime}$, induced by $(\cdot)^{b}$. A similar notation will be later used for any other Euclidean vector space.

Definition 2.1. The special T-algebra determined by $\left(V, g_{V}\right)$ and $\left(S=S_{0}+S_{1}, g_{S}\right)$ is the direct sum of vector spaces

$$
\mathcal{A}=\left(S_{0}^{\prime}+V^{\prime}+S_{1}^{\prime}\right)+\mathbb{R}+\mathbb{R}+\mathbb{R}+\left(V+S_{1}+S_{0}\right),
$$

equipped with the product "." and the Euclidean scalar product $(\cdot, \cdot)$ defined as follows. Consider the notation

$$
\begin{array}{lll} 
& \mathcal{A}_{11}=\mathcal{A}_{22}=\mathcal{A}_{33}:=\mathbb{R}, & \\
\mathcal{A}_{12}:=V, & \mathcal{A}_{23}:=S_{0}, & \mathcal{A}_{13}:=S_{1}, \\
\mathcal{A}_{21}:=\mathcal{A}_{12}^{\prime}=V^{\prime}, & \mathcal{A}_{32}:=\mathcal{A}_{23}^{\prime}=S_{0}^{\prime}, & \mathcal{A}_{31}:=\mathcal{A}_{13}^{\prime}=S_{1}^{\prime}
\end{array}
$$

and define "." as the product such that the only non-trivial multiplications are given by the bilinear maps $\cdot: \mathcal{A}_{i j} \times \mathcal{A}_{j \ell} \longrightarrow \mathcal{A}_{i \ell}$ that are determined by the Clifford multiplication $\mu: V \times S \rightarrow S$ as follows:

$$
\begin{array}{rlrl}
\cdot & : \mathcal{A}_{i i} \times \mathcal{A}_{i \ell} \rightarrow \mathcal{A}_{i \ell}, & \rho \cdot a & =\rho a \quad \text { (mult. by the real number } \rho), \\
\cdot & : \mathcal{A}_{i j} \times \mathcal{A}_{j i} \rightarrow \mathcal{A}_{i i}, & x \cdot y^{b} & =y \cdot x^{b}:=\langle x, y\rangle \quad \text { (scalar product) }, \\
\cdot & : \mathcal{A}_{12} \times \mathcal{A}_{23} \rightarrow \mathcal{A}_{13}, & v \cdot s_{0}:=\mu\left(v, s_{0}\right) \quad \text { (Clifford multiplication), } \\
\cdot: \mathcal{A}_{31} \times \mathcal{A}_{12} \rightarrow \mathcal{A}_{32}, & \left\langle v^{b} \cdot s_{1}, s_{0}\right\rangle:=\left\langle\mu\left(v, s_{0}\right), s_{1}\right\rangle \text { (dual Cliff. mult.), } \\
\cdot: \mathcal{A}_{23} \times \mathcal{A}_{31} \rightarrow \mathcal{A}_{21}, & \left\langle s_{0} \cdot s_{1}^{b}, v^{b}\right\rangle:=\left\langle\mu\left(v, s_{0}\right), s_{1}\right\rangle,
\end{array}
$$

together with the rules

$$
\begin{array}{rlrl}
\cdot: \mathcal{A}_{i j} \times \mathcal{A}_{j j} & \rightarrow \mathcal{A}_{i j}, & a_{i j} \cdot \rho:=\rho \cdot a_{i j}, & \\
\cdot: \mathcal{A}_{i j} \times \mathcal{A}_{j \ell} \rightarrow \mathcal{A}_{i \ell}, & a_{i j} \cdot a_{j \ell}:=\left(a_{j \ell}^{*} \cdot a_{i j}^{*}\right)^{*}, & i<j<\ell
\end{array}
$$

where $(\cdot)^{*}: \mathcal{A} \rightarrow \mathcal{A}$ is the involutive linear map such that $\mathcal{A}_{i j}^{*}=\mathcal{A}_{j i}$ with

$$
\left.(\cdot)^{*}\right|_{\mathcal{A}_{i j}}= \begin{cases}\operatorname{Id}_{\mathcal{A}_{i i}} & \text { if } i=j, \\ (\cdot)^{b} & \text { if } i<j, \\ (\cdot)^{\#} & \text { if } i>j .\end{cases}
$$

This completely determines all of the remaining products.

Finally, we set $(\cdot, \cdot)$ to be the Euclidean scalar product on $\mathcal{A}$, with respect to which all subspaces $\mathcal{A}_{i j}$ are orthogonal one to the other, is the standard scalar product of $\mathbb{R}$ on each subspace $\mathcal{A}_{11}=\mathcal{A}_{22}=\mathcal{A}_{33}=\mathbb{R}$, and is equal to $\langle\cdot, \cdot\rangle_{V^{\prime}+S^{\prime}}+\langle\cdot, \cdot\rangle$ on the subspace $\left(S_{0}^{\prime}+V^{\prime}+S_{1}^{\prime}\right)+\left(V+S_{1}+S_{0}\right)$. 
In [2] it is proven that any special $T$-algebra, as defined above, satisfies the axioms of $T$-algebras of a rank 3 in the sense of Vinberg. This can be also directly checked, by observing that the Lie algebra

$$
\mathfrak{N}=\mathcal{A}_{12}+\mathcal{A}_{13}+\mathcal{A}_{23}=V+S_{0}+S_{1},
$$

equipped with the Euclidean scalar product $(\cdot, \cdot)_{\mathcal{N} \times \mathcal{N}}$, is an $N$-algebra of rank 3 in the sense of [40, section III.7]. Indeed, by Vinberg's results, any $T$-algebra of rank 3 is uniquely determined by its nilpotent part $\left(\mathfrak{N}=\mathcal{A}_{12}+\mathcal{A}_{23}+\mathcal{A}_{13},(\cdot, \cdot)\right)$, which is required just to be associative and with isometric product $\cdot: \mathcal{A}_{12} \times \mathcal{A}_{23} \rightarrow \mathcal{A}_{13}$.

The subsets of $\mathcal{A}=\mathcal{A}(V, S)$, defined by

$$
\begin{aligned}
G & :=\left\{x=\left(x_{1}, x_{2}, x_{3}\right)+\boldsymbol{v}+\boldsymbol{s}_{0}+\boldsymbol{s}_{1} \in \mathbb{R}^{3}+V+S_{0}+S_{1}, x_{i}>0\right\}, \\
G^{*} & :=\left\{y=\boldsymbol{s}_{0}{ }^{*}+\boldsymbol{v}^{*}+\boldsymbol{s}_{1}{ }^{*}+\left(y_{1}, y_{2}, y_{3}\right) \in S_{0}^{\prime}+V^{\prime}+S_{1}^{\prime}+\mathbb{R}^{3}, y_{i}>0\right\}
\end{aligned}
$$

are closed under the multiplication - and such a product defines the structure of a simply connected solvable Lie group on each of them.

Definition 2.2. The group $G$ is called Vinberg triangular group and $G^{*}$ is called dual (triangular) Vinberg group.

By the results in [40], $G$ and $G^{*}$ are both simply connected and solvable and the map $\imath: G \rightarrow G^{*}, \imath(A):=\left(A^{*}\right)^{-1}$, is a Lie group isomorphism.

\subsubsection{The standard matrix representation of a special $T$ algebra}

Let

$$
\mathcal{A}=\left(S_{0}^{\prime}+V^{\prime}+S_{1}^{\prime}\right)+\mathbb{R}^{3}+\left(V+S_{0}+S_{1}\right)
$$

be the special T-algebra $\mathcal{A}=\mathcal{A}(V, S)$, determined by a Euclidean vector space $\left(V, g_{V}\right)$ and a metric $\mathbb{Z}_{2}$-graded $\mathcal{C} \ell(V)$-module $\left(S=S_{0}+S_{1}, g_{S}\right)$. Each element $x=\sum_{i, j=1}^{3} x_{i j}$ of $\mathcal{A}=\sum_{i, j=1}^{3} \mathcal{A}_{i j}$ is representable by the $3 \times 3$ matrix

$$
X(x)=\left(\begin{array}{lll}
x_{11} & x_{12} & x_{13} \\
x_{21} & x_{22} & x_{23} \\
x_{31} & x_{32} & x_{33}
\end{array}\right)=\left(\begin{array}{ccc}
x_{1} & v & s_{1} \\
w^{*} & x_{2} & s_{0} \\
t_{1}^{*} & t_{0}^{*} & x_{3}
\end{array}\right),
$$

where $x_{i}=x_{i i} \in \mathcal{A}_{i i}=\mathbb{R}$ and

$$
\begin{array}{lll}
x_{12}=v \in \mathcal{A}_{12}=V, & x_{13}=s_{1} \in \mathcal{A}_{13}=S_{1}, & x_{23}=s_{0} \in \mathcal{A}_{23}=S_{0}, \\
x_{21}=w^{*} \in \mathcal{A}_{21}=V^{\prime}, & x_{31}=t_{1}^{*} \in \mathcal{A}_{31}=S_{1}^{\prime}, & x_{32}=t_{0}^{*} \in \mathcal{A}_{32}=S_{0}^{\prime} .
\end{array}
$$

The product $\cdot$ of $\mathcal{A}$ defines the (non-associative) product between these matrices, determined by the standard matrix multiplication,

$$
\left(x_{i j}\right) \cdot\left(y_{\ell m}\right):=\left(z_{i m}=\sum_{j=1}^{3} x_{i j} \cdot y_{j m}\right) .
$$


Since this matrix product satisfies

$$
X(x) \cdot X(y)=X(x \cdot y), \quad \text { for any } x, y \in \mathcal{A},
$$

the linear map

$$
\mathcal{A} \longrightarrow \operatorname{Mat}=\operatorname{Mat}(V, S):=\{X:=X(x), x \in \mathcal{A}\}, \quad x \longmapsto X(x),
$$

is a linear representation, called the (standard) matrix representation of $\mathcal{A}$.

In terms of the matrix representation $\operatorname{Mat}=\operatorname{Mat}(V, S)$, the involution $(\cdot)^{*}$ of the $T$-algebra $\mathcal{A}$ is given by

$$
X^{*}=\left(\begin{array}{ccc}
x_{1} & v & s_{1} \\
w^{*} & x_{2} & s_{0} \\
t_{1}^{*} & t_{0}^{*} & x_{3}
\end{array}\right)^{*}:=\left(\begin{array}{ccc}
x_{1} & w & t_{1} \\
v^{*} & x_{2} & t_{0} \\
s_{1}^{*} & s_{0}^{*} & x_{3}
\end{array}\right) .
$$

Using this map, we can express the scalar product $(\cdot, \cdot)$ of $\mathcal{A}$ in terms the matrix representation by the formula

$$
(x, y)=\operatorname{tr}\left(X(x) \cdot Y^{*}(y)\right) .
$$

The space of Hermitian matrices in Mat is the subspace defined by

$$
\mathcal{H}:=\left\{X \in \text { Mat }: X=X^{*}\right\} .
$$

It has a natural algebra structure determined by the Jordan multiplication

$$
X \circ Y:=\frac{1}{2}(X Y+Y X) .
$$

Definition 2.3. The commutative algebra $\mathcal{H}$ is called the Hermitian Vinberg algebra associated with the metric $\mathcal{C} \ell(V)$-module $\left(S, g_{S}\right)$.

In the matrix representation, the (upper) triangular group $G$ and the dual (lower) triangular group $G^{*}$ are represented by the non-degenerate upper triangular matrices

$$
A=\left(\begin{array}{ccc}
\boldsymbol{\rho}_{1} & \boldsymbol{v} & \boldsymbol{s}_{1} \\
0 & \boldsymbol{\rho}_{2} & \boldsymbol{s}_{0} \\
0 & 0 & \boldsymbol{\rho}_{3}
\end{array}\right), \quad \boldsymbol{\rho}_{i}>0, \boldsymbol{v} \in V, \boldsymbol{s}_{0} \in S_{0}, \boldsymbol{s}_{1} \in S_{1}
$$

and the lower triangular matrices

$$
B=\left(\begin{array}{ccc}
\boldsymbol{\tau}_{1} & 0 & 0 \\
\boldsymbol{w}^{*} & \boldsymbol{\tau}_{2} & 0 \\
\boldsymbol{t}_{1}^{*} & \boldsymbol{t}_{0}^{*} & \boldsymbol{\tau}_{3}
\end{array}\right), \quad \boldsymbol{\tau}_{i}>0, \boldsymbol{w} \in V, \boldsymbol{t}_{0} \in S_{0}, \boldsymbol{t}_{1} \in S_{1},
$$

respectively. The isomorphism $\imath: G \rightarrow G^{*}, \imath(A)=A^{-1 *}$ is explicitly given by

$$
A=\left(\begin{array}{ccc}
\rho_{1} & \boldsymbol{v} & \boldsymbol{s}_{1} \\
0 & \boldsymbol{\rho}_{2} & \boldsymbol{s}_{0} \\
0 & 0 & \boldsymbol{\rho}_{3}
\end{array}\right) \longrightarrow A^{-1 *}=\left(\begin{array}{ccc}
\frac{1}{\rho_{1}} & 0 & 0 \\
-\frac{\boldsymbol{v}^{*}}{\rho_{1} \rho_{2}} & \frac{1}{\rho_{2}} & 0 \\
-\frac{\left(\rho_{2} s_{1}-\boldsymbol{v} \cdot s_{0}\right)^{*}}{\rho_{1} \rho_{2} \rho_{3}} & -\frac{s_{0}^{*}}{\rho_{2} \rho_{3}} & \frac{1}{\rho_{3}}
\end{array}\right)
$$

The Lie algebra $\mathfrak{g}$ (resp. $\mathfrak{g}^{*}$ ) of the group $G$ (resp. $G^{*}$ ) consists of all upper triangular (resp., lower triangular) matrices in Mat $\simeq \mathcal{A}$. The groups of upper and lower triangular matrices (2.14) and (2.15) are called standard realisations of $G$ and $G^{*}$, respectively. 


\subsection{Special Vinberg cones and their dual cones}

\subsubsection{The linear actions of $G$ and $G^{*}$ on the space of Hermitian matrices $\mathcal{H}$}

As we mentioned above, the Lie algebras $\mathfrak{g}=\operatorname{Lie}(G)$ and $\mathfrak{g}^{*}=\operatorname{Lie}\left(G^{*}\right)$ consist of arbitrary upper and lower triangular matrices in Mat $\simeq \mathcal{A}$. As it can be directly checked using the axioms of $T$-algebras, the formula

$$
T_{B} X:=B \cdot X+X \cdot B^{*}, \quad X \in \mathcal{H}
$$

defines two linear representations $T: \mathfrak{g} \times \mathcal{H} \rightarrow \mathcal{H}$ and $T: \mathfrak{g}^{*} \times \mathcal{H} \rightarrow \mathcal{H}$ of these Lie algebras on the vector space $\mathcal{H}$. Since the Lie groups $G, G^{*}$ are solvable and simply connected, these Lie algebras representations integrate to linear representations of the groups $G$ and $G^{*}$ given by

$$
\begin{aligned}
\exp (B)(X):= & \exp \left(T_{B}\right) X=X+T_{B} X+\frac{1}{2} T_{B}^{2} X+\ldots \\
= & X+B \cdot X+X \cdot B^{*}+ \\
& +\frac{1}{2}\left(B \cdot(B \cdot X)+B \cdot\left(X \cdot B^{*}\right)+(B \cdot X) \cdot B^{*}+\left(X \cdot B^{*}\right) \cdot B^{*}\right)+\ldots
\end{aligned}
$$

Due to the non-associativity of the algebra $\mathcal{A}$, in general this action of the elements $A=\exp (B)$ in $G$ or $G^{*}$ on the elements $X \in \mathcal{H}$ cannot be reduced to the standard expression $A \cdot X \cdot A^{*}$.

\subsubsection{The linear representation of $G$ on the dual space $\mathcal{H}^{\prime}=\operatorname{Hom}(\mathcal{H}, \mathbb{R})$}

Given the vector space of Hermitian matrices $\mathcal{H} \subset$ Mat, we use the Euclidean metric $\langle X, Y\rangle:=\operatorname{tr} X \cdot Y$ of $\mathcal{H}$ to identify the dual vector space $\mathcal{H}^{\prime}=\operatorname{Hom}(\mathcal{H}, \mathbb{R})$ with $\mathcal{H}$. More precisely, any $X \in \mathcal{H}$ is identified with the 1 -form $X^{b}=\langle X, \cdot\rangle$.

The elements $A$ of the group $G$ act on $\mathcal{H}^{\prime}$ by the dual transformations

$$
A\left(X^{b}\right)(Y):=X^{*}\left(A^{-1}(Y)\right)=\operatorname{tr}\left(X \cdot\left(A^{-1}(Y)\right), \quad Y \in \mathcal{H} .\right.
$$

We denote by $G^{\prime}$ the group of these dual transformations. It is the exponential of the dual action of the elements $B$ of the Lie algebra $\mathfrak{g}=\operatorname{Lie}(G)$ given by

$$
T_{B}\left(X^{b}\right)=\left(-T_{B^{*}}(X)\right)^{b}=-\left(B^{*} \cdot X+X \cdot B\right)^{b} .
$$

Indeed, for any $Y \in \mathcal{H}$,

$$
\begin{aligned}
T_{B}\left(X^{b}\right)(Y)=-\operatorname{tr}\left(X \cdot(B \cdot Y)+X \cdot\left(Y \cdot B^{*}\right)\right) & \text { Ax. III \& Ax. IV of } T \text {-algebras } \\
=-\operatorname{tr}((X \cdot B) \cdot Y)) & -\operatorname{tr}\left(\left(B^{*} \cdot X\right) \cdot Y\right)= \\
& =-\operatorname{tr}\left(T_{B^{*}}(X) \cdot Y\right)=\left(-T_{B^{*}}(X)\right)^{b}(Y) .
\end{aligned}
$$

Exponentiating both sides of (2.18), it follows that for any $A=\exp (B) \in G$,

$$
A\left(X^{b}\right)=\exp \left(T_{B}\right)\left(X^{b}\right)=\left(\exp \left(T_{-B^{*}}\right)(X)\right)^{b}=\left(A^{-1 *}(X)\right)^{b} .
$$

Hence, under the above identification $\mathcal{H}^{\prime} \simeq \mathcal{H}$, the group $G^{\prime}$ acting on $\mathcal{H}^{\prime}$ corresponds to the group $G^{*}=\left\{\imath(A)=A^{-1 *}, A \in G\right\}$ acting on $\mathcal{H}$. 


\subsubsection{The special Vinberg cones and their dual and adjoint cones}

By Vinberg's results in [40] the following holds.

Theorem 2.4. The orbits $\mathcal{V}=G(I)$ and $\mathcal{V}^{*}=G^{*}(I)$ of the identity matrix $I \in \mathcal{H}$ are equal to

$$
\mathcal{V}=\left\{A \cdot A^{*}, A \in G\right\}, \quad \mathcal{V}^{*}=\left\{A^{*} \cdot A, A \in G\right\} .
$$

They are both homogeneous convex cones, on which the groups $G$ and, respectively, $G^{*}$ act simply transitively.

Definition 2.5. The convex cones $\mathcal{V}=G(I)$ and $\mathcal{V}^{*}=G^{*}(I)$ are called special Vinberg cone and its dual cone, respectively, associated with the metric $\mathbb{Z}_{2}$-graded $\mathcal{C} \ell(V)$-module $\left(S, g_{S}\right)$.

The dual cone $\mathcal{V}^{*}$ has the following important geometric role. Consider the adjoint cone of the cone $\mathcal{V} \subset \mathcal{H}$, that is the cone in $\mathcal{H}^{\prime} \simeq \mathcal{H}$ defined by

$$
\mathcal{V}^{\prime}=\{X \in \mathcal{H}: \operatorname{tr}(X \cdot Y)>0, Y \in \overline{\mathcal{V}} \backslash\{0\}\} .
$$

It can be proved that $\mathcal{V}^{\prime}=\mathcal{V}^{*}=G^{*}(I)$. We finally recall the following

Definition 2.6. A homogeneous cone $\mathcal{V} \subset \mathcal{H}$ is called self-adjoint or symmetric if there exists a vector space isomorphism $L: \mathcal{H} \rightarrow \mathcal{H}$ such that $L(\mathcal{V})=\mathcal{V}^{*}$.

\subsection{Group coordinates, adapted orthogonal coordinates and de Wit and Van Proeyen coordinates}

Since both cones $\mathcal{V}=G(I)$ and $\mathcal{V}^{*}=G^{*}(I)$ are in natural bijection with $G$, we may consider the diffeomorphisms $\xi_{G}: \mathcal{V} \rightarrow G$ and $\xi_{G}^{*}: \mathcal{V}^{*} \rightarrow G$ given by

$$
X=A \cdot A^{*} \stackrel{\xi_{G}}{\longmapsto} A, \quad Y=A^{-1 *} \cdot A^{-1} \stackrel{\xi_{G}^{*}}{\longmapsto} A .
$$

We call them the group coordinates of $\mathcal{V}$ and $\mathcal{V}^{*}$.

The relations between the group coordinates and the corresponding elements in $\mathcal{V}$ and $\mathcal{V}^{*}$ are as follows. If $X \in \mathcal{V}$ corresponds to the element $A=\left(\begin{array}{ccc}\rho_{1} & v & s_{1} \\ 0 & \rho_{2} & s_{0} \\ 0 & 0 & \rho_{3}\end{array}\right) \in G$, than the entries of $X=\left(\begin{array}{ccc}x_{1} & v & s_{1} \\ v^{b} & x_{2} & s_{0} \\ s_{1}^{b} & s_{0}^{b} & x_{3}\end{array}\right)=A \cdot A^{*}$ are

$$
\begin{aligned}
& x_{1}=\boldsymbol{\rho}_{1}^{2}+|\boldsymbol{v}|^{2}+\left|s_{1}\right|^{2}, \quad x_{2}=\boldsymbol{\rho}_{2}^{2}+\left|s_{0}\right|^{2}, \quad x_{3}=\boldsymbol{\rho}_{3}^{2}, \\
& v=\rho_{2} \boldsymbol{v}+s_{1} \cdot s_{0}^{b}, \quad s_{0}=\rho_{3} s_{0}, \quad s_{1}=\rho_{3} s_{1} \text {. }
\end{aligned}
$$

Similarly, if $X \in \mathcal{V}^{*}$ corresponds to $\xi_{G}^{*}(X)=A=\left(\begin{array}{ccc}\rho_{1} & \boldsymbol{v} & s_{1} \\ 0 & \rho_{2} & s_{0} \\ 0 & 0 & \rho_{3}\end{array}\right)$, the entries of $X=$ $A^{-1 *} \cdot A^{-1}$ are

$$
\begin{aligned}
& x_{1}=\frac{1}{\boldsymbol{\rho}_{1}^{2}}, \quad x_{2}=\frac{\left(\boldsymbol{\rho}_{1} \boldsymbol{\rho}_{3}\right)^{2}+\boldsymbol{\rho}_{3}^{2}|\boldsymbol{v}|^{2}}{\left(\boldsymbol{\rho}_{1} \boldsymbol{\rho}_{2} \boldsymbol{\rho}_{3}\right)^{2}}, \quad x_{3}=\frac{\left|\boldsymbol{\rho}_{2} \boldsymbol{s}_{1}-\boldsymbol{v} \cdot \boldsymbol{s}_{0}\right|^{2}+\boldsymbol{\rho}_{1}^{2}\left|\boldsymbol{s}_{0}\right|^{2}+\boldsymbol{\rho}_{1}^{2} \boldsymbol{\rho}_{2}^{2}}{\left(\boldsymbol{\rho}_{1} \boldsymbol{\rho}_{2} \boldsymbol{\rho}_{3}\right)^{2}}, \\
& v=-\frac{\boldsymbol{\rho}_{2} \boldsymbol{\rho}_{3}^{2} \boldsymbol{v}}{\left(\boldsymbol{\rho}_{1} \boldsymbol{\rho}_{2} \boldsymbol{\rho}_{3}\right)^{2}}, \quad s_{0}=\frac{\boldsymbol{\rho}_{3} \boldsymbol{v}^{\mathrm{b}} \cdot\left(\boldsymbol{\rho}_{2} \boldsymbol{s}_{1}-\boldsymbol{v} \cdot \boldsymbol{s}_{0}\right)+\boldsymbol{\rho}_{1}^{2} \boldsymbol{\rho}_{3} \boldsymbol{s}_{0}}{\left(\boldsymbol{\rho}_{1} \boldsymbol{\rho}_{2} \boldsymbol{\rho}_{3}\right)^{2}}, \quad s_{1}=\frac{-\boldsymbol{\rho}_{2} \boldsymbol{\rho}_{3}\left(\boldsymbol{\rho}_{2} \boldsymbol{s}_{1}-\boldsymbol{v} \cdot \boldsymbol{s}_{0}\right)}{\left(\boldsymbol{\rho}_{1} \boldsymbol{\rho}_{2} \boldsymbol{\rho}_{3}\right)^{2}} .
\end{aligned}
$$


The inverses to the (2.22) are given by (see also $[1,40]$ )

$$
\begin{aligned}
\boldsymbol{\rho}_{3}^{2} & =x_{3}, \\
\boldsymbol{\rho}_{2}^{2} & =x_{2}-\frac{1}{\boldsymbol{\rho}_{3}^{2}}\left|s_{0}\right|^{2}=\frac{x_{3} x_{2}-\left|s_{0}\right|^{2}}{x_{3}}, \\
\boldsymbol{\rho}_{1}^{2} & =\frac{x_{1} x_{2} x_{3}-x_{1}\left|s_{0}\right|^{2}-x_{2}\left|s_{1}\right|^{2}-x_{3}|v|^{2}+2\left(v, s_{1} \cdot s_{0}^{*}\right)}{\left(\boldsymbol{\rho}_{2} \boldsymbol{\rho}_{3}\right)^{2}}= \\
& =\frac{x_{1} x_{2} x_{3}-x_{1}\left|s_{0}\right|^{2}-x_{2}\left|s_{1}\right|^{2}-x_{3}|v|^{2}+2\left\langle\mu\left(v, s_{0}\right), s_{1}\right\rangle}{x_{3} x_{2}-\left|s_{0}\right|^{2}}, \\
\boldsymbol{v} & =\frac{1}{\boldsymbol{\rho}_{2}} v-\frac{1}{\boldsymbol{\rho}_{2} \boldsymbol{\rho}_{3}^{2}} s_{1} \cdot s_{0}^{b}, \quad \boldsymbol{s}_{0}=\frac{1}{\boldsymbol{\rho}_{3}} s_{0}, \quad \boldsymbol{s}_{1}=\frac{1}{\boldsymbol{\rho}_{3}} s_{1},
\end{aligned}
$$

while the inverses to the (2.23) are given by

$$
\begin{aligned}
\frac{1}{\boldsymbol{\rho}_{1}^{2}} & =x_{1} \\
\frac{1}{\boldsymbol{\rho}_{2}^{2}} & =x_{2}-\boldsymbol{\rho}_{1}^{2}|v|^{2}=\frac{x_{1} x_{2}-|v|^{2}}{x_{1}}, \\
\frac{1}{\boldsymbol{\rho}_{3}^{2}} & =\left(\boldsymbol{\rho}_{1} \boldsymbol{\rho}_{2}\right)^{2}\left(x_{1} x_{2} x_{3}-x_{1}\left|s_{0}\right|^{2}-x_{2}\left|s_{1}\right|^{2}-x_{3}|v|^{2}+2\left\langle v^{b} \cdot s_{1}, s_{0}\right\rangle\right)= \\
& =\frac{x_{1} x_{2} x_{3}-x_{1}\left|s_{0}\right|^{2}-x_{2}\left|s_{1}\right|^{2}-x_{3}|v|^{2}+2\left\langle\mu\left(v, s_{0}\right), s_{1}\right\rangle}{x_{1} x_{2}-|v|^{2}}, \\
\boldsymbol{v} & =-\boldsymbol{\rho}_{1}^{2} \boldsymbol{\rho}_{2} v, \quad \boldsymbol{s}_{0}=-\boldsymbol{\rho}_{1}^{2} \boldsymbol{\rho}_{2}^{2} \boldsymbol{\rho}_{3} v^{b} \cdot s_{1}+\boldsymbol{\rho}_{2}^{2} \boldsymbol{\rho}_{3} s_{0}, \\
\boldsymbol{\rho}_{2} \boldsymbol{s}_{1} & =\boldsymbol{v} \cdot \boldsymbol{s}_{0}-\frac{\left(\boldsymbol{\rho}_{1} \boldsymbol{\rho}_{2} \boldsymbol{\rho}_{3}\right)^{2}}{\boldsymbol{\rho}_{2} \boldsymbol{\rho}_{3}} s_{1} .
\end{aligned}
$$

Note that (2.24) imply that $\mathcal{V}$ (resp. (2.25) imply that $\mathcal{V}^{*}$ ) coincides with the convex set characterised by the following three inequalities

$$
\begin{aligned}
& x_{3}>0, \quad x_{3} x_{2}-\left|s_{0}\right|^{2}>0, \\
& x_{1} x_{2} x_{3}-x_{1}\left|s_{0}\right|^{2}-x_{2}\left|s_{1}\right|^{2}-x_{3}|v|^{2}+2\left\langle\mu\left(v, s_{0}\right), s_{1}\right\rangle>0, \\
\text { (resp. } & x_{1}>0, \quad x_{1} x_{2}-|v|^{2}>0 \\
& \left.x_{1} x_{2} x_{3}-x_{1}\left|s_{0}\right|^{2}-x_{2}\left|s_{1}\right|^{2}-x_{3}|v|^{2}+2\left\langle\mu\left(v, s_{0}\right), s_{1}\right\rangle>0\right)
\end{aligned}
$$

which generalise Sylvester's criterion for positive definiteness.

We now observe that $\mathcal{H}$ is isomorphic (as a vector space) to the vector space $W=$ $\mathbb{R}^{3}+V+S_{0}+S_{1}=\mathcal{A}_{11}+\mathcal{A}_{22}+\mathcal{A}_{33}+V+S_{0}+S_{1}$. Therefore each basis $\mathcal{B}$ for $W$ naturally determines an associated system of coordinates for $\mathcal{H} \simeq W$, which we call standard. If $\mathcal{B}$ has the form

$$
\mathcal{B}=\left(\mathbf{1}_{1}, \mathbf{1}_{2}, \mathbf{1}_{3}, e_{j}, f_{0 \mid \alpha}, f_{1 \mid \beta}, f_{0 \mid \alpha}^{*}, f_{1 \mid \beta}^{*}\right),
$$


with $\mathbf{1}_{i}$ standard basis of $\mathcal{A}_{i i}=\mathbb{R}$ and $\left(e_{j}\right),\left(f_{0 \mid \alpha}, f_{1 \mid \beta}\right)$ orthonormal bases of $\left(V, g_{V}\right)$ and $\left(S=S_{0}+S_{1}, g_{S}\right)$, respectively, the corresponding coordinates on $\mathcal{H}$

$$
X=\left(\begin{array}{ccc}
y^{1} \mathbf{1}_{1}=x_{1} & v^{i} e_{i} & s_{1}^{\beta} f_{1 \mid \beta} \\
v^{i} f_{i}^{*} & y^{2} \mathbf{1}_{2}=x_{2} & s_{0}^{\alpha} f_{0 \mid \alpha} \\
\left(s_{1}^{\beta} f_{1 \mid \beta}\right)^{*} & \left(s_{0}^{\alpha} f_{0 \mid \alpha}\right)^{*} & y^{3} \mathbf{1}_{3}=x_{3}
\end{array}\right) \stackrel{\mathcal{B}}{\longrightarrow}\left(\begin{array}{c}
y=\left(y^{i}\right) \\
v=\left(v^{j}\right) \\
s_{0}=\left(s_{0}^{\alpha}\right) \\
s_{1}=\left(s_{1}^{\beta}\right)
\end{array}\right)
$$

are called adapted orthogonal coordinates on $\mathcal{H}$. A basis $\mathcal{B}^{\prime}$ for the space $W^{\prime}=\operatorname{Hom}(\mathcal{W}, \mathbb{R})$, which is dual to a basis $\mathcal{B}$ for $W$, determines coordinates for $\mathcal{H}^{\prime} \simeq W^{\prime}$, which we call dual coordinates associated with $\mathcal{B}$.

For a given system of adapted orthogonal coordinates

$$
\left(y^{1}, y^{2}, y^{3}, v=\left(v^{j}\right), s_{0}=\left(s_{0}^{\alpha}\right), s_{1}=\left(s_{1}^{\beta}\right)\right),
$$

the associated de Wit-Van Proeyen coordinates are the coordinates $w=\left(w^{I}\right)$ determined by the linear transformation rules

$$
\begin{aligned}
& w^{1}=y^{3}, \quad w^{2}=\frac{y^{1}+y^{2}}{2}, \quad w^{3}=\frac{-y^{1}+y^{2}}{2}, \quad w^{\widetilde{\mu}}=v^{\widetilde{\mu}-3}, \\
& w^{i}=s_{0}^{i-(3+n)}, \quad w^{i^{\prime}}=s_{1}^{i^{\prime}-\left(3+n+\frac{n_{S}}{2}\right)},
\end{aligned}
$$

with $4 \leq \widetilde{\mu} \leq n+3,4+n \leq i \leq 4+n+\frac{n_{S}}{2}$ and $4+n+\frac{n_{S}}{2} \leq i^{\prime} \leq 4+n+n_{S}$, where $n:=\operatorname{dim} V$ and $n_{S}=\operatorname{dim} S=\operatorname{dim} S_{0}+\operatorname{dim} S_{1}=2 \operatorname{dim} S_{0}$. The corresponding dual transformation rules

$$
\begin{aligned}
& w_{1}=y_{3}, \quad w_{2}=y_{1}+y_{2}, \quad w_{3}=-y_{1}+y_{2}, \quad w_{\widetilde{\mu}}=v_{\widetilde{\mu}-3}, \\
& w_{i}=s_{0 \mid i-(3+n)}, \quad w_{i^{\prime}}=s_{1 \mid i^{\prime}-\left(3+n+\frac{n_{S}}{2}\right)},
\end{aligned}
$$

define the De Wit-Van Proeyen dual coordinates.

\subsection{The invariant and dual invariant cubic polynomials}

The Lie group $G$ of upper triangular matrices is the direct product $G=\left(\mathbb{R}_{+} I\right) \times G_{0}$ of the dilatation subgroup $\mathbb{R}_{+} I$ and the unimodular subgroup

$$
G_{0}:=\left\{A=\left(\begin{array}{ccc}
\rho_{1} & \boldsymbol{v} & \boldsymbol{s}_{1} \\
0 & \boldsymbol{\rho}_{2} & \boldsymbol{s}_{0} \\
0 & 0 & \boldsymbol{\rho}_{3}
\end{array}\right), \boldsymbol{\rho}_{1} \boldsymbol{\rho}_{2} \boldsymbol{\rho}_{3}=1\right\} .
$$

Similarly $G^{*}$ is the direct product $G^{*}=\left(\mathbb{R}_{+} I\right) \times G_{0}^{*}$ of the dilatation subgroup $\mathbb{R}_{+} I$ and the unimodular subgroup $G_{0}^{*} \subset G^{*}=\imath(G)$

$$
G_{0}^{*}:=\left\{B=\left(\begin{array}{ccc}
\boldsymbol{\tau}_{1} & 0 & 0 \\
\boldsymbol{u}^{b} & \boldsymbol{\tau}_{2} & 0 \\
\boldsymbol{t}_{1}^{b} & \boldsymbol{t}_{0}^{b} & \boldsymbol{\tau}_{3}
\end{array}\right), \boldsymbol{\tau}_{1} \boldsymbol{\tau}_{2} \boldsymbol{\tau}_{3}=1\right\}=\imath\left(G_{0}\right)
$$


Definition 2.7. A non-zero polynomial $p: \mathcal{H} \rightarrow \mathbb{R}$ is called invariant cubic (resp. dual invariant cubic) if

$$
p(t A(X))=t^{3} p(X) \quad \text { with } t \in \mathbb{R}_{+}, A \in G_{0} \quad\left(\text { resp. } A \in G_{0}^{*}\right) .
$$

Theorem 2.8. Up to a scaling factor, there exist a unique invariant cubic polynomial $d: \mathcal{H} \rightarrow \mathbb{R}$ and a unique dual invariant cubic polynomial $d^{*}: \mathcal{H} \rightarrow \mathbb{R}$. Assuming the normalising condition $d(I)=d^{*}(I)=1$, these two cubic polynomials are equal and both given by

$$
d(X)=d^{*}(X)=x_{1} x_{2} x_{3}-x_{1}\left|s_{0}\right|^{2}-x_{2}\left|s_{1}\right|^{2}-x_{3}|v|^{2}+2\left(v \cdot s_{0}\right) \cdot s_{1}^{b} .
$$

Proof. The invariant and dual invariant cubic polynomials are unique up to a scaling because the subgroups $G_{0}$ and $G_{0}^{*}$ have codimension one orbits. We now prove that, if $d(I)=d^{*}(I)=1$, then $d(X)=d^{*}(X)$ and that the formula for $d(X)$ is (2.33). By real analyticity, it suffices to prove this for $X=A \cdot A^{*} \in G(I)$ and $X^{*}=A^{*} \cdot A \in G^{*}(I)$ for some $A=\left(\begin{array}{ccc}\rho_{1} & \boldsymbol{v} & s_{1} \\ 0 & \rho_{2} & s_{0} \\ 0 & 0 & \rho_{3}\end{array}\right)$.

The squared determinant map on $G$ and the inverse of the square determinant map on $G^{*}$, i.e. the functions

$$
(\operatorname{det} A)^{2}:=\left(\boldsymbol{\rho}^{1} \boldsymbol{\rho}^{2} \boldsymbol{\rho}^{3}\right)^{2}=\left(a_{11} a_{22} a_{33}\right)^{2}, \frac{1}{(\operatorname{det} A)^{2}}:=\frac{1}{\left(\boldsymbol{\rho}^{1} \boldsymbol{\rho}^{2} \boldsymbol{\rho}^{3}\right)^{2}}=\frac{1}{\left(a_{11} a_{22} a_{33}\right)^{2}},
$$

are $G_{0}$-invariant and $G_{0}^{*}$-invariant, respectively, and they are both equal to 1 on $A=I$. They are therefore the unique $G_{0^{-}}$and $G_{0^{*}}^{*}$ invariant functions $d: \mathcal{V} \rightarrow \mathbb{R}$ and $d^{*}: \mathcal{V}^{*} \rightarrow \mathbb{R}$ satisfying the normalising condition. The inverse map (2.24) and (2.25) show that the expressions of these two function in terms of the entries of the matrix $X$ are both equal to $(2.33)$.

In adapted orthogonal coordinates the invariant polynomial (2.33) is

$$
d\left(y, v, s_{0}, s_{1}\right)=d^{*}\left(y, v, s_{0}, s_{1}\right)=y^{1} y^{2} y^{3}-y^{1}\left|s_{0}\right|^{2}-y^{2}\left|s_{1}\right|^{2}-y^{3}|v|^{2}+2 \gamma_{i \alpha \beta} v^{i} s_{0}^{\alpha} s_{1}^{\beta},
$$

where $\gamma_{i \alpha \beta}$ is the $\Gamma$-matrix representing the Clifford multiplication $\mu\left(e_{i}, \cdot\right)$. Let us now denote by $\breve{\gamma}_{3}=\left(\breve{\gamma}_{3 \mathbf{i j}}\right)$ and $\check{\gamma}_{\widetilde{\mu}}=\left(\check{\gamma}_{\widetilde{\mu}_{\mathbf{i j}}}\right)$ the square matrices, acting on the $n_{S^{-} \text {-dimensional }}$ space $S=S_{0}+S_{1}$ (whose elements have components denoted by $\left(s^{\mathbf{i}}\right)=\left(s_{0}^{\alpha}, s_{1}^{\beta}\right)$ for short) given by

$$
\check{\gamma}_{3}=\left(\begin{array}{cc}
I_{\frac{n_{S}}{2}} & 0 \\
0 & -I_{\frac{n_{S}}{2}}
\end{array}\right), \quad \check{\gamma}_{\widetilde{\mu}}:=\left(\begin{array}{cc}
0 & \gamma_{\widetilde{\mu}-3} \\
\gamma_{\widetilde{\mu}-3} & 0
\end{array}\right) .
$$

By the transformation rules (2.30), the expression for $d=d^{*}$ in de Wit-Van Proeyen coordinates $w=\left(w^{I}\right)=\left(w^{1}, w^{2}, w^{3}, w^{\widetilde{\mu}}, w^{\mathbf{i}}\right)$ becomes

$$
\begin{gathered}
d\left(w^{I}\right)=d^{*}\left(w^{I}\right)=w^{1}\left(w^{2}\right)^{2}-w^{1}\left(w^{3}\right)^{2}-w^{1} \delta_{\tilde{\mu \nu}} w^{\widetilde{\mu}} w^{\widetilde{\nu}}-w^{2} \delta_{i j} w^{\mathbf{i}} w^{\mathbf{j}}+\breve{\gamma}_{3 \mathbf{i}} w^{3} w^{\mathbf{i}} w^{\mathbf{j}}+\check{\gamma}_{\mu \mathbf{i} \mathbf{j}} w^{\widetilde{\mu}} w^{\mathbf{i}} w^{\mathbf{j}}= \\
=w^{1}\left(w^{2}\right)^{2}-w^{1} \delta_{\mu \nu} w^{\mu} w^{\nu}-w^{2} \delta_{\mathbf{i} \mathbf{j}} w^{\mathbf{i}} w^{\mathbf{j}}+\breve{\gamma}_{\mu \mathbf{i} \mathbf{j}} w^{\mu} w^{\mathbf{i}} w^{\mathbf{j}},
\end{gathered}
$$


where $\mu$ runs between 3 and $3+n$ and $\mathbf{i}, \mathbf{j}$ run between $4+n$ and $3+n+n_{S} \cdot{ }^{1}$ Note that

- the $\breve{\gamma}_{\mu}$ are the Dirac matrices of the representation of the Clifford algebra $\mathcal{C} \ell\left(V^{\prime}, g_{V^{\prime}}\right)$, $V^{\prime}:=\mathbb{R} \oplus V, g_{V^{\prime}}:=-d t^{2} \oplus g_{V}$, on the spinor space $S=S_{0}+S_{1}$;

- up to the factor 3, (2.35) is the formula found by de Wit and Van Proeyen in their classification of invariant cubic polynomials ([18, formula (4.2)]).

This motivates the name of the coordinates $\left(w^{I}\right)$, one of the most frequently used set of coordinates in the physics literature on invariant cubic polynomials.

A non-zero polynomial $p^{\prime}: \mathcal{H}^{\prime} \rightarrow \mathbb{R}$ is called invariant dual cubic polynomial if $p^{\prime}\left(t A^{\prime}(X)\right)=t^{3} p^{\prime}(X)$ for any $t A \in G=\left(\mathbb{R}_{+} I\right) \times G_{0}$.

The isomorphism $(\cdot)^{b}: \mathcal{H} \rightarrow \mathcal{H}^{\prime}$ maps each invariant cubic polynomial $d$ on $\mathcal{H}$ onto a uniquely associated invariant dual cubic polynomial $d^{\prime}$ on $\mathcal{H}^{\prime}$. Thus, theorem 2.8 implies that up to a scaling, there is a unique invariant dual cubic polynomial $d^{\prime}: \mathcal{H}^{\prime} \rightarrow \mathbb{R}$, which is determined as follows. Given an adapted system of coordinates $x=\left(x^{a}\right)$ on $\mathbb{R}^{n}=\mathcal{H}$, if we denote by $g=\left(g_{\text {oab }}\right)$ the components of the Euclidean scalar product $\langle\cdot, \cdot\rangle$ in the coordinate basis, by $d_{a b c}$ the (symmetric in all indices) coefficients of the cubic polynomial $d(x)=d^{*}(x)=d_{a b c} x^{a} x^{b} x^{c}$ and by $g_{o}^{-1}:=\left(g_{o}^{a b}\right)$ the inverse of $g_{o}$, then $d^{\prime}(x)=$ $g_{o}^{a a^{\prime}} g_{o}^{b b^{\prime}} g_{o}^{c c^{\prime}} d_{a^{\prime} b^{\prime} c^{\prime}} x_{a} x_{b} x_{c}$.

This means that, if the coordinates are orthogonal (thus with $\left.g_{o}=\left(g_{o a b}\right)=\left(\delta_{a b}\right)\right)$ and the components of $d$ in these coordinates are denoted by $d_{a b c}$, then the components $d^{a b c}$ of the dual polynomial $d^{\prime}$ in the associated dual adapted orthogonal coordinates are obtained by simply raising the indices with the matrix $\delta^{a b}$. In particular, if the coordinates are not just orthogonal, but also adapted (i.e. as in (2.29)), then the expression for $d^{\prime}$ is

$$
d^{\prime}\left(y, v^{b}, s_{0}^{b}, s_{1}^{b}\right)=y_{1} y_{2} y_{3}-y_{1}\left|s_{0}^{b}\right|^{2}-y_{2}\left|s_{1}^{b}\right|^{2}-y_{3}\left|v^{b}\right|^{2}+2 \gamma^{i \alpha \beta} v_{i} s_{0 \alpha} s_{1 \beta} .
$$

On the other hand, since the de Wit-Van Proeyen coordinates $\left(w^{I}\right)$ are obtained from the adapted orthogonal coordinates $\left(y^{a}, v^{j}, s_{0}^{\alpha}, s_{1}^{\beta}\right)$ by means of the non-orthogonal transformation (2.30), the entries of the matrix $\left(g_{o I J}\right)$ and of its inverse in such new coordinates are

$$
\left(g_{o I J}\right)=\left(\begin{array}{cccc}
1 & 0 & 0 & 0 \\
0 & 2 & 0 & 0 \\
0 & 0 & 2 & 0 \\
0 & 0 & 0 & I_{n+n_{S}}
\end{array}\right), \quad\left(g_{o}^{I J}\right)=\left(\begin{array}{cccc}
1 & 0 & 0 & 0 \\
0 & \frac{1}{2} & 0 & 0 \\
0 & 0 & \frac{1}{2} & 0 \\
0 & 0 & 0 & I_{n+n_{S}}
\end{array}\right) .
$$

Hence the expression for $d^{\prime}$ in the de Wit-Van Proeyen coordinates is

$$
d^{\prime}\left(w_{I}\right)=\frac{1}{4} w_{1} w_{2}^{2}-\frac{1}{4} w_{1} w_{3}^{2}-w_{1} \delta^{\widetilde{\mu \nu}} w_{\widetilde{\mu}} w_{\widetilde{\nu}}-\frac{1}{2} w_{2} \delta^{\mathbf{i} \mathbf{j}} w_{\mathbf{i}} w_{\mathbf{j}}+\frac{1}{2} \widetilde{\gamma}_{3}^{\mathbf{i} \mathbf{j}} w_{3} w_{\mathbf{i}} w_{\mathbf{j}}+\widetilde{\gamma}^{\widetilde{\mu} \mathbf{j} \mathbf{j}} w_{\widetilde{\mu}} w_{\mathbf{i}} w_{\mathbf{j}},
$$

where $\widetilde{\mu}$ runs between 4 and $3+n$ and $\mathbf{i}, \mathbf{j}$ run between $4+n$ and $3+n+n_{S}$.

\footnotetext{
${ }^{1}$ Mind the differences in indices conventions: on one hand in adapted orthogonal coordinates we denote the vector (resp. spinor) indices by Latin letters as $i, j$ (resp. Greek letters like $\alpha, \beta$ ); on the other hand, following a traditional choice of physics literature, in the de Wit-Van Proeyen coordinates the vector (resp. spinor) indices are denoted by Greek letters of the sequence $\mu, \nu$, etc. (resp. Latin letters $\mathbf{i}, \mathbf{j}$ and so on).
} 


\subsection{Quadratic maps associated with invariant cubic polynomials and their inverses}

In what follows, we identify any cubic polynomial $d: \mathcal{H} \rightarrow \mathbb{R}$ and any dual cubic polynomial $d^{\prime}: \mathcal{H}^{\prime} \rightarrow \mathbb{R}$ with the associated symmetric tenors $d \in S^{3} \mathcal{H}^{\prime}$ and $d^{\prime} \in S^{3}\left(\mathcal{H}^{\prime}\right)^{\prime}=S^{3} \mathcal{H}$ determined by polarisation.

Given a special Vinberg cone $\mathcal{V} \subset \mathcal{H}$, we denote by $d_{v}$ and $d_{v}^{\prime}$ the uniquely associated invariant cubic and invariant dual cubic polynomials, defined by (2.33) and (2.36), respectively (i.e. the unique invariant polynomials satisfying the normalising conditions $d_{v}(I)=1$ and $\left.d_{v}^{\prime}\left(I^{b}\right)=1\right)$. By theorem 2.8 all other invariant cubic and invariant dual cubic polynomials associated with $\mathcal{V}$ have the forms

$$
d=k d_{\nu} \quad \text { with } k:=d(I), \quad d^{\prime}=k^{\prime} d_{v} \quad \text { with } k^{\prime}:=d^{\prime}\left(I^{b}\right) .
$$

With no loss of generality from now on we assume $k>0$ and $k^{\prime}>0$. Actually, given $d=k d_{\mathcal{V}}$, we constantly denote by $d^{\prime}$ the canonically associated dual polynomial, which is defined by

$$
d^{\prime}:=\frac{1}{k} d_{v}^{\prime} .
$$

As we announced in the introduction, the problem of determining the entropy of extremal BPS black holes in the supergravity theories of this paper can be reduced to the mathematical question of finding the inverses of a certain quadratic maps determined by cubic polynomials (see [26]). In the cases in which the scalar manifold is a homogeneous space determined by an irreducible cubic polynomial, such a cubic polynomial is the invariant polynomial $d$ associated with an appropriate special Vinberg cone. In these cases, the quadratic map that needs to be inverted is defined as follows.

Definition 2.9. Given a invariant cubic polynomial $d=k d_{v}$ and its canonically associated dual polynomial $d^{\prime}=\frac{1}{k} d_{v}^{\prime}$, the associated quadratic maps are the maps $h, h^{\prime}$ from $\mathcal{H}$ to $\mathcal{H}^{\prime}=\operatorname{Hom}(\mathcal{H}, \mathbb{R})$ and vice versa defined by

$$
h(X):=d(X, X, \cdot) \quad h^{\prime}\left(X^{b}\right):=d^{\prime}\left(X^{b}, X^{b}, \cdot\right) .
$$

The next lemma and theorem solve the mentioned inversion problem and leads to the solution to our BPS black hole entropy problem under the assumption that the scalar manifold is homogeneous, but not necessarily symmetric (see section 5.4).

Lemma 2.10. Let $\mathcal{V} \subset \mathcal{H}$ be a special Vinberg cone with associated $d=k d_{\mathcal{V}}, d^{\prime}=\frac{1}{k} d_{\mathcal{V}}^{\prime}$ and with $h, h^{\prime}$ corresponding quadratic maps, as defined above. Let also denote

$$
\begin{aligned}
& \mathcal{H}_{+}:=\{d>0\}, \quad \mathcal{H}_{-}:=\{d<0\} \quad \subset \mathcal{H}, \\
& \mathcal{H}_{+}^{\prime}:=\left\{d^{\prime}>0\right\}, \quad \mathcal{H}_{-}^{\prime}:=\left\{d^{\prime}<0\right\} \subset \mathcal{H}^{\prime} .
\end{aligned}
$$

(i) The maps $h$ and $h^{\prime}$ are $G_{0}$-equivariant with respect to the natural $G_{0}$-actions on $\mathcal{H}$ and $\mathcal{H}^{\prime}=\operatorname{Hom}(\mathcal{H}, \mathbb{R})$, i.e.

$$
h(A(X))=A^{\prime}(h(X)) \quad \text { and } \quad h^{\prime}\left(A^{\prime}\left(X^{b}\right)\right)=A\left(h^{\prime}\left(X^{b}\right)\right) .
$$

Hence $h$ and $h^{\prime}$ map any $G$-orbit in $\mathcal{H}$ into a $G$-orbit in $\mathcal{H}^{\prime}$. 
(ii) $h(I)=k I^{\mathrm{b}}$ and $h^{\prime}\left(I^{\mathrm{b}}\right)=\frac{1}{k} I$.

(iii) for any $X \in \mathcal{H}$

$$
\left(h^{\prime} \circ h\right)(X)=d(X) X \quad\left(h \circ h^{\prime}\right)\left(X^{b}\right)=d^{\prime}\left(X^{b}\right) X^{b} .
$$

(iv) The map $h\left(\right.$ resp. $\left.h^{\prime}\right)$ determines a $G_{0}$-equivariant diffeomorphism from each $G$-orbit in $\mathcal{H}_{+} \cup \mathcal{H}_{-}$(resp. $\left.\mathcal{H}_{+}^{\prime} \cup \mathcal{H}_{-}^{\prime}\right)$ into a G-orbit in $\mathcal{H}_{+}^{\prime}$ (resp. in $\mathcal{H}_{+}$) and it maps $\mathcal{H}_{0}:=\{d=0\}$ into $\mathcal{H}_{0}^{\prime}:=\left\{d^{\prime}=0\right\}$ (resp. $\mathcal{H}_{0}^{\prime}$ into $\mathcal{H}_{0}$ ); in particular the maps $h: \mathcal{V} \rightarrow \mathcal{V}^{\prime}$ and $h^{\prime}: \mathcal{V}^{\prime} \rightarrow \mathcal{V}$ are diffeomorphisms.

(v) Each map $h: \mathcal{H}_{ \pm} \rightarrow \mathcal{H}_{+}^{\prime}$ (resp. $h^{\prime}: \mathcal{H}_{ \pm}^{\prime} \rightarrow \mathcal{H}_{+}$) is a diffeomorphism onto $\mathcal{H}_{+}^{\prime} \subset \mathcal{H}^{\prime}$ (resp. onto $\left.\mathcal{H}_{+} \subset \mathcal{H}\right)$.

Proof.

(i) It suffices to observe that

$$
\begin{aligned}
h(A(X))(Y) & =d(A(X), A(X), Y)= \\
& =d\left(A(X), A(X), A\left(A^{-1}(Y)\right)\right)=d\left(X, X, A^{-1}(Y)\right)=A^{\prime}(h(X))(Y) .
\end{aligned}
$$

(ii) It is a direct consequence of

$$
h(I)(Y)=k d_{\mathcal{v}}(I, I, Y)=k \operatorname{Tr}(Y)=k \operatorname{Tr}(I \cdot Y)=k I^{\mathrm{b}}(Y) .
$$

(iii) We first prove the first identity of (2.42) in case $X \in \mathcal{V}$, i.e. under the assumption that $X=t A(I)$ for some $t \in \mathbb{R}_{+}$and $A \in G_{0}$. Then

$$
\left(h^{\prime} \circ h\right)(X)=h^{\prime}(h(t A(I)))=t^{4} h^{\prime}\left(k A^{\prime}\left(I^{b}\right)\right)=k^{2} t^{3}\left(t A h^{\prime}\left(I^{b}\right)\right)=t^{3} k(t A(I))=d(X) X .
$$

A similar argument proves the second identity in case $X^{b} \in \mathcal{V}^{\prime}$. By real analyticity, the two identities of (2.42) holds for any $X \in \mathcal{H}$.

(iv) Let $d(X) \neq 0$ and $X^{\prime}:=h(X)$. By (2.42) we have $h^{\prime}\left(X^{\prime}\right)=d(X) X$ and therefore

$$
d^{\prime}\left(X^{\prime}\right)=h^{\prime}\left(X^{\prime}\right)\left(X^{\prime}\right)=d(X) X^{\prime}(X)=d(X) h(X)(X)=d(X)^{2} \neq 0 .
$$

This shows that $h\left(\mathcal{H}_{ \pm}\right) \subset \mathcal{H}_{+}^{\prime}$ and, by a similar argument, that $h^{\prime}\left(\mathcal{H}_{ \pm}^{\prime}\right) \subset \mathcal{H}_{+}$. The claim then follows from (i) and (iii).

(v) For the maps $h: \mathcal{H}_{ \pm} \rightarrow \mathcal{H}_{+}^{\prime}$, it suffices to observe that for any $h(X) \in \mathcal{H}_{+}^{\prime}$ with $X \in \mathcal{H}_{ \pm}$

$$
\frac{1}{\sqrt{d^{\prime}(h(X))}} h^{\prime}(h(X)) \stackrel{(2.42) \stackrel{\&}{=}}{(2.43)} \frac{1}{\sqrt{(d(X))^{2}}} d(X) X= \pm X .
$$

A similar argument shows the existence of the inverse for $h^{\prime}: \mathcal{H}_{ \pm}^{\prime} \rightarrow \mathcal{H}_{+}$. 
The previous lemma and (2.44) imply the following

Theorem 2.11. The inverse map $h^{-1}$ of the diffeomorphism $h: \mathcal{H}_{+} \rightarrow \mathcal{H}_{+}^{\prime}$ (resp. $h$ : $\mathcal{H}_{-} \rightarrow \mathcal{H}_{+}^{\prime}$ ) is given by

$$
h^{-1}\left(X^{b}\right)=\frac{1}{\sqrt{d^{\prime}\left(X^{b}\right)}} h^{\prime}\left(X^{b}\right) \quad\left(\operatorname{resp} . h^{-1}\left(X^{b}\right):=-\frac{1}{\sqrt{d^{\prime}\left(X^{b}\right)}} h^{\prime}\left(X^{b}\right)\right) .
$$

Remark 2.12. If the special Vinberg cone $\mathcal{V}$ is symmetric (see definition 2.6), the relation (2.42) corresponds to the adjoint identity of its canonically associated cubic Jordan algebra (see e.g. [33, section 3.8]). Denoting by $d_{a b c}$ and $d^{\prime d e f}$ the tensorial components of $d$ and $d^{\prime}$, respectively, in a system of standard coordinates $\left(y^{a}\right)$ for $\mathcal{H}$, it takes the polarised form (up to a normalisation) found in supergravity literature (see e.g. $[9,15,27]$ and references therein)

$$
d^{\prime a b c} d_{a(d f \mid} d_{b \mid g h)}=\delta_{(d}^{c} d_{f g h)}
$$

\section{Projective-special Kähler manifolds and very special cones}

The scalar manifolds (i.e. the target spaces of the maps representing the scalar field sector of the Maxwell-Einstein 4-dimensional supergravity theories considered in this paper) are Kähler manifolds of a particular kind, the so-called projective-special Kähler manifolds. For reader's convenience, we briefly review some properties of these manifolds and of their fundamental relations with the special Vinberg cones (for further information, see [11-13]; see also the extensive discussion in [30] and references therein).

\subsection{Conical scalar manifolds, projective scalar manifolds and special Vinberg cones}

A conical affine special Kähler (for short, conical scalar) manifold is a Kähler manifold $\left(\mathcal{M}, J, g_{\mathcal{N}}\right)$ equipped with a flat torsion free connection $\nabla$ and a homothetic vector field $\xi$ such that:

(i) $\nabla\left(g_{\mathcal{M}} \circ J\right)=0$;

(ii) $\left(\nabla_{X} J\right) Y=\left(\nabla_{Y} J\right) X$;

(iii) $\nabla \xi=D \xi=$ Id where $D$ is the Levi-Civita connection of $\left(\mathcal{M}, g_{\mathcal{M N}}\right)$;

(iv) the metric $g_{\mathcal{M}}$ is positively defined on $\mathcal{D}:=\operatorname{span}(\xi, J \xi)$ and it is negatively defined on $\mathcal{D}^{\perp}$;

(v) the commuting holomorphic vector fields $\xi, J \xi$ are complete and define a free holomorphic $\mathbb{C}^{*}$-action

$$
\left(\rho e^{i \vartheta}, x\right) \longmapsto t e^{i \vartheta} \cdot x:=\left(e^{t \xi} \circ e^{\vartheta J \xi}\right)(x),
$$

in which $\left\{x \mapsto e^{i \vartheta} \cdot x=e^{\vartheta J \xi}(x)\right\}$ is a one-parameter isometry group. 
In this case the hypersurface

$$
\widetilde{\mathcal{S}}:=\left\{x \in \mathcal{M},\left.\quad g_{\mathcal{M}}(\xi, \xi)\right|_{\mathcal{M}}=1\right\} \subset \mathcal{M}
$$

is $S^{1}=\left\{e^{i \theta}\right\}$-invariant, with an induced Lorentzian metric $\left.g\right|_{T \widetilde{\mathcal{S}}}$ of signature $(1,2 n)$ and a sub-Riemannian structure $\left(\langle J \xi\rangle^{\perp},-\left.g_{\widetilde{\mathcal{S}}}\right|_{\left\langle\langle J \xi\rangle^{\perp}\right.}\right)$ of rank $2 n$, which are both preserved by the $S^{1}$-action. By $S^{1}$-invariance, the sub-Riemannian structure $\left(\langle J \xi\rangle^{\perp},-\left.g_{\mathfrak{\mathcal { S }}}\right|_{\left\langle\langle J \xi\rangle^{\perp}\right.}\right)$ projects onto a Kähler structure $\left(J, g_{\mathcal{S}}\right)$ on the quotient $\mathcal{S}=\widetilde{\mathcal{S}} / S^{1} \simeq \mathcal{M} / \mathbb{C}^{*}([3])$.

The Kähler manifolds $\left(\mathcal{S}, J, g_{\mathcal{S}}\right)$ determined in this way are called projective special Kähler manifolds or, motivated by physics terminology, projective scalar manifolds.

\subsection{Description of projective scalar manifolds in terms of conical holomorphic coordinates and prepotentials}

Let $\left(\left(\mathcal{M}, J, g_{\mathcal{M}}\right), \nabla, \xi\right)$ be a conical scalar manifold (= conical affine special Kähler manifold) of $\operatorname{dim}_{\mathbb{R}} \mathcal{M}=2 n+2$ and with associated projective scalar $2 n$-manifold $\left(\mathcal{S}, J, g_{\mathcal{S}}\right)$. Around any point of $\mathcal{M}$, there exists a neighbourhood $\mathcal{U} \subset \mathcal{M}$, on which there exist a distinguished system of holomorphic coordinates $X=\left(X^{I}\right), I=0,1, \cdots, n$, called conical special coordinates, and a homogeneous of degree 2 holomorphic function $F: \mathcal{U} \rightarrow \mathbb{C}$, called prepotential, which locally determines all data of $\mathcal{M}$ as follows.

(a) the complex structure $J$ is given by the multiplication by $i=\sqrt{-1}$ on the holomorphic vector fields $\frac{\partial}{\partial X^{I}}$;

(b) $g_{\mathcal{M}}$ is the metric given by

$$
g_{\mathcal{M}}=m_{I J} d X^{I} \otimes d \bar{X}^{J} \text { with } m_{I J}(X, \bar{X}):=2 \operatorname{Im}\left(F_{I J}\right)=\left.2 \operatorname{Im}\left(\frac{\partial^{2} F}{\partial X^{I} \partial X^{J}}\right)\right|_{(X, \bar{X})}
$$

and has the real function $\varphi=-\log \left(\left.g_{\mathcal{M}}(\xi, \xi)\right|_{\mathfrak{U}}\right)$ as Kähler potential;

(c) $\xi$ is the vector field $\xi=X^{I} \frac{\partial}{\partial X^{I}}+\bar{X}^{I} \frac{\partial}{\partial \bar{X}^{I}}$;

(d) using the notation $x^{I}:=\operatorname{Re}\left(X^{I}\right), y^{J}:=\operatorname{Im}\left(X^{J}\right)$ and $y_{J}:=\operatorname{Re}\left(F_{J}\right)$, the connection $\nabla$ is uniquely determined by the conditions that $x^{I}$ and $y_{J}$ are $\nabla$-flat, i.e. $\nabla \frac{\partial}{\partial x^{I}}=$ $\nabla \frac{\partial}{\partial y_{J}}=0$. Note that, since $y_{J} \neq y^{J}$, in general the vector fields $\frac{\partial}{\partial X^{I}}=\frac{\partial}{\partial x^{I}}-i \frac{\partial}{\partial y^{I}}$ are not $\nabla$-flat.

The $\mathbb{C}^{*}$-invariant functions $z^{a}=\frac{X^{a}}{X^{0}}, 1 \leq a \leq n$, are holomorphic coordinates on the corresponding open subset $\widetilde{U} \subset \mathcal{U} / \mathbb{C}^{*}$ in the projective-special Kähler manifold $(\mathcal{S}=$ $\left.\mathcal{M} / \mathbb{C}^{*}, J, g_{\mathcal{S}}\right)$ and any $\mathbb{C}^{*}$-invariant map $f: \mathcal{U} \rightarrow \mathbb{R}$ corresponds to a unique function $f_{\widetilde{U}}: \widetilde{U} \subset \mathcal{S} \rightarrow \mathbb{R}$ given by

$$
\left.f_{\widetilde{u}}\right|_{z=[X]}=f\left(1, X^{1}, \cdots, X^{n}\right)
$$

(in other words, in physics jargon, the Kähler gauge freedom is fixed such that $X^{0}=1$ ). In the open subset $\widetilde{\mathcal{U}} \subset \mathcal{S}$, the metric $g_{\mathcal{S}}$ has the Kähler potential

$$
\left.\mathcal{K}\right|_{z=\left[1: X^{1}: \ldots: X^{n}\right]_{\mathbb{C}^{*}}}:=-\log \sum_{I, J=0}^{n} X^{I} m_{I J}(X, \bar{X}) \bar{X}^{J} .
$$




\subsection{The supergravity r-map}

Let $W=\mathbb{R}^{n+1}$ be an $n+1$-dimensional vector space. A homogeneous cubic polynomial $d: W \rightarrow \mathbb{R}$ is called hyperbolic if the Hessian $\partial^{2} d_{x}$ has signature $(1, n)$ at some $x \in W$. A hypersurface $\mathcal{T} \subset W$ is called very special real if there exists a homogeneous cubic polynomial $d$ such that $\mathcal{T} \subset \mathcal{T}_{d}=\{d=1\}$ and the tensor field $g_{\mathcal{T}}:=-\left.\partial^{2} d\right|_{T \mathcal{T}}$ is a Riemannian metric on $\mathcal{T}$. Note that if this occurs, then $d$ is hyperbolic.

The supergravity $r$-map is the correspondence which associates with each very special real manifold $\left(\mathcal{T}, g_{\mathcal{T}}\right)$ the projective scalar manifold $\left(\mathcal{S}_{\mathcal{T}}, J, g_{\mathcal{S}_{\mathcal{T}}}\right)$ where: (i) $\mathcal{S}_{\mathcal{T}}$ is the open submanifold

$$
\mathcal{S}_{\mathcal{T}}:=\mathbb{R}^{n}+i \mathcal{V} \subset \mathbb{C}^{n}, \quad \mathcal{V}=\mathbb{R}_{+} \cdot \mathcal{T},
$$

called Siegel domain, and (ii) $J$ is the standard complex structure of $\mathbb{C}^{n}$ and (iii) $g_{\mathcal{S}_{\mathcal{J}}}$ is the Kähler metric, given in terms of the standard coordinates $\left(z^{a}=x^{a}+i y^{a}\right)$ on $\mathbb{C}^{n}$ by

$$
g_{\mathcal{S}_{\mathcal{T}}}=\partial_{z^{a}} \partial_{z^{b}} \mathcal{K}(z, \bar{z}) d z^{a} \otimes d \bar{z}^{b}, \quad \mathcal{K}:=-\log 8 d(y) .
$$

The triple $\left(\mathcal{S}_{\mathcal{T}}, J, g_{\mathcal{S}_{\mathcal{T}}}\right)$ is the projectivisation of the conical scalar manifold

$$
\mathcal{M}_{\mathcal{T}}:=\left\{X=X^{0} \cdot\left(1, z^{1}, \ldots, z^{n}\right), \quad X^{0} \in \mathbb{C}^{*}, z \in \mathcal{S}=\mathbb{R}^{n}+i \mathcal{V}\right\} \subset \mathbb{C}^{n+1}
$$

with Kähler metric, homothetic vector field and connection $\nabla$, which are uniquely determined by the coordinates

$$
\left(X^{I}\right)=\left(X^{0}, X^{1}=X^{0} \cdot z^{1}, \ldots, X^{n}=X^{0} \cdot z^{n}\right)
$$

of $\mathbb{C}^{n+1}$ and the prepotential

$$
F: \mathcal{M}_{\mathcal{T}} \longrightarrow \mathbb{C}, \quad F\left(X^{I}\right):=\frac{d\left(X^{1}, X^{2}, \ldots, X^{n}\right)}{X^{0}}
$$

as in (a)-(d) of section 3.2.

The projective scalar manifold $\left(\mathcal{S}_{\mathcal{T}}=\mathcal{M}_{\mathcal{T}} / \mathbb{C}^{*}, J, g_{\mathcal{S}_{\mathcal{T}}}\right)$ is the image of the special cubic $\left(\mathcal{T}, g_{\mathcal{T}}\right)$ under the $r$-map. Note that, up to local equivalences, the $r$-map is essentially surjective, namely

Theorem 3.1 ([12, proposition $1.6 \& 1.10])$. Any $2 n$-dimensional projective scalar manifold $\left(\mathcal{S}, J, g_{\mathcal{S}}\right)$ is locally isometrically biholomorphic to the image under the $r$-map $\left(\mathcal{S}_{\mathcal{T}}, J, g_{\mathcal{S}_{\mathcal{T}}}\right)$ of a special cubic $\left(\mathcal{T}, g_{\mathcal{T}}\right)$ in $W=\mathbb{R}^{n}$.

If the special cubic cone $\mathcal{V}=\mathbb{R}_{+} \cdot \mathcal{T}$ of a special cubic $\left(\mathcal{T}, g_{\mathcal{T}}\right) \subset \mathbb{R}^{n}$ is convex and homogeneous, the special cubic manifold $\left(\mathcal{T}, g_{\mathcal{T}}\right)$ is called Vinberg cubic.

Theorem $3.2([\mathbf{1 2}, \mathbf{1 8}])$. Let $\left(\mathcal{T}, g_{\mathcal{T}}\right)$ be a special cubic manifold associated with an irreducible polynomial d (that is, not decomposable as the product of two lower degree polynomials). The manifold $\mathcal{T}$ is a Vinberg cubic if and only if the corresponding cone $\mathcal{V}=\mathbb{R}_{+} \cdot \mathcal{T}$ is a special Vinberg cone and $\mathcal{T}=\{d=1\} \cap \mathcal{V}$. 


\section{BPS black holes in $\mathcal{N}=24 D$ supergravity}

\subsection{Scalar fields, vector fields and central charges in an $\mathcal{N}=24 D$ supergravity theory}

As we mentioned in the introduction, our discussion is focused on the BPS black holes in theories of ungauged $\mathcal{N}=2$ supergravity on a 4-dimensional Lorentzian space-time $M$. For comprehensive overviews of the contents of such supergravity theories we refer to the foundational papers $[14,16,17]$ and to the vast subsequent literature on the topic (see e.g. $[4,30]$ and references therein). For our purposes we need to know just a few basic features, which we now recall.

For what concerns the physical contents of such supergravity theories, we are interested only in the gravitational field (i.e. a Lorentzian metric $g$ on the space-time $M$ ) and in the bosonic sector of the vector multiplets, namely:

- the scalar fields, which are mathematically represented by the coordinate components of a map $z: M \rightarrow \mathcal{S}$ into an appropriate target complex $n$-manifold $\mathcal{S}$, called scalar manifold;

- the vector fields of $n+1$ abelian gauge fields; up to gauge transformations these fields are given by the components $A_{\mu}^{I}, 0 \leq I \leq n$, of the potential of a connection on a principal bundle $\pi: P \rightarrow M$ of the abelian group $T^{n+1}=\underbrace{S^{1} \times \ldots \times S^{1}}_{n+1 \text { times }}$.

The scalar manifold $\mathcal{S}$ is always assumed to be a projective scalar manifold $(\mathcal{S}=$ $\left.\mathcal{S}_{\mathcal{T}}, J, g_{\mathcal{S}}\right)$ of complex dimension $n$ which is image of a special cubic $\left(\mathcal{T}, g_{\mathcal{T}}\right)$ under the $r$ map, as defined in section 3.1. Such a scalar manifold $\mathcal{S}=\mathcal{S}_{\mathcal{T}}$ is therefore a complex manifold of the form $\mathcal{S}=\mathbb{R}^{n}+i \mathcal{V}$ where $\mathcal{V}=\mathbb{R}_{+} \cdot \mathcal{T} \subset\{d>0\}$ is a special cubic cone associated with $\mathcal{T} \subset\{d=1\} \subset \mathbb{R}^{n}$. By theorem 3.2, if $d$ is irreducible and $\mathcal{V}$ is convex and homogeneous, then $\mathcal{V}$ is a special Vinberg cone according to the definition in section 2.2.

The field strengths of the abelian gauge fields (= the electromagnetic fields) of the theory are geometrically given by the $n+1$ components of the curvature 2 -form

$$
\mathbb{F}=\left(\mathbb{F}_{\mu \nu}^{0} d y^{\mu} \wedge d y^{\nu}, \ldots, \mathbb{F}_{\mu \nu}^{n} d y^{\mu} \wedge d y^{\nu}\right), \quad \text { with } \quad \mathbb{F}_{\mu \nu}^{I}:=\frac{1}{2}\left(\frac{\partial A_{\mu}^{I}}{\partial y^{\nu}}-\frac{\partial A_{\nu}^{I}}{\partial y^{\mu}}\right),
$$

where $\left(y^{\mu}\right)$ are coordinates for the space-time $M$.

In addition to the scalar and the abelian gauge fields, there is another important function which has to be considered: the central charge $Z$. It is a $\mathbb{C}$-valued field $Z: M \rightarrow \mathbb{C}$ on the space-time which is determined by the physical fields of the theory and satisfies a continuity equation.

The notion of the central charge was first introduced in [37, 41] for generic solutions of supersymmetric theories and gives important information on the physical properties of the solutions of the field equations. For an extensive discussion, we refer the interested reader to the original papers. For our purposes, we need to recall just a couple of facts concerning its relation with the entropy of the black holes. We briefly review them in section 4.3. 


\subsection{Static and spherically symmetric black holes and their electro-magnetic charges}

From now on, we assume to be working in a fixed supergravity theory on a 4-dimensional space time of the kind described in section 4.1. Moreover, by "black hole" we constantly understand a static, spherically symmetric and asymptotically flat gravity field that solves the equations of the theory and has the singularity of an isolated black hole. In mathematical terms this means that the metric $g$ and the bosonic fields associated with the black hole satisfy the following conditions.

(i) The Lorentzian manifold $(M, g)$ admits a set of globally defined coordinates $(t, r, \vartheta, \varphi)$, where:

(a) the vector field $\frac{\partial}{\partial t}$ is time-like,

(b) each level set $M_{t=t_{o}}=\left\{t=t_{o}\right\}$ is space-like and diffeomorphic to $\mathbb{R}^{3}$,

(c) $(r, \vartheta, \varphi)$ are spherical coordinates for each submanifold $M_{t=t_{o}} \simeq \mathbb{R}^{3}$.

(ii) The metric has the form

$$
g=-e^{2 U(r)} d t^{2}+e^{-2 U(r)}\left(d r^{2}+r^{2}\left(d \theta^{2}+\sin ^{2} \theta d \varphi^{2}\right)\right)
$$

such that $g$ tends to the flat metric for $r \rightarrow \infty$, and

$$
e^{-2 U(r)} \cong_{r \rightarrow 0^{+}} \frac{C^{2}}{r^{2}}, \quad \text { for some constant } C \in \mathbb{R},
$$

so that there is an event horizon at $r=0$ (see for instance [5, 21]). The geometry in the near-horizon limit $r \rightarrow 0^{+}$is the Bertotti-Robinson geometry $A d S_{2} \times S^{2}[8,36]$. The area of the $S^{2}$ which equals the area of the unique event horizon reads

$$
A_{H}=4 \pi C^{2} \text {. }
$$

(iii) The scalar fields and the electromagnetic fields are $t$-independent and invariant under the standard $\mathrm{SO}_{3}$-action on each space-like submanifold $M_{t=t_{o}} \simeq \mathbb{R}^{3}$.

By saying that "there is a black hole horizon at $r=0$ " we mean that the value $r=0$ determines the boundary of an $\mathrm{SO}_{3}$-invariant and time independent region of the space-like manifold $M_{t=t_{o}} \simeq \mathbb{R}^{3}$, from which no light ray might escape.

Given a black hole, for any fixed values $t_{o}$ and $r_{o} \mathfrak{g} 0$, we may consider the double integrals

$$
\begin{aligned}
p^{I} & :=\frac{1}{4 \pi} \int_{\mathbf{S}=\left\{t=t_{o}, r=r_{o}\right\}} \mathbb{F}^{I} \\
& =\frac{1}{4 \pi} \iiint_{\vartheta \in[0, \pi], \varphi \in[0,2 \pi]} \mathbb{F}_{\vartheta \varphi}^{I} d \vartheta \wedge d \varphi, \quad \text { with } \quad \mathbb{F}_{\vartheta \varphi}^{I}:=\left.\mathbb{F}^{I}\right|_{\mathbf{S}}\left(\frac{\partial}{\partial \vartheta}, \frac{\partial}{\partial \varphi}\right), \\
q_{I} & :=\frac{1}{4 \pi} \int_{\vartheta \in[0, \pi], \varphi \in[0,2 \pi]} \mathbb{G}_{I \mid \vartheta \varphi} d \vartheta \wedge d \varphi, \quad \text { with } \quad \mathbb{G}_{I \mid \vartheta \varphi}:=\left(\left.\star \mathbb{F}^{I}\right|_{\mathbf{S}}\right)\left(\frac{\partial}{\partial \vartheta}, \frac{\partial}{\partial \varphi}\right),
\end{aligned}
$$


where $\mathbb{G}$ is a tensor field which is uniquely determined by the electromagnetic field $\mathbb{F}$ by means of an appropriate generalised Hodge star operator. This operator depends in a nontrivial way on the scalar fields, but for our purposes we do not need to know its explicit expression. By (iii) and Gauss' theorem, these integrals are independent on the choice of $t_{o}$ and $r_{o}$ and can be considered as the sources (= "charges") of the $n+1$ electromagnetic fields $\mathbb{F}^{I}:=\mathbb{F}_{\mu \nu}^{I} d y^{\mu} \wedge d y^{\nu}$ at large distances from the black hole. Indeed the 2-forms $\mathbb{F}^{I}$ behave precisely as if they were classical electromagnetic fields, generated by corresponding magnetic monopoles and/or electric charges, all of them located at $r=0$. The values $p^{I}, q_{I}$ are called the magnetic charges and the electric charges of the black hole, respectively. If there are two charges $p^{I}, q_{I}$, which are both non trivial, the corresponding electromagnetic field $\mathbb{F}^{I}$ behaves as if it were generated by a dyon and the black hole is called dyonic.

\subsection{BPS black holes, their entropy and the "inverse relation" map}

The first of the two properties of the central charge, which we need to recall, is that for each black hole satisfying (i), (ii), (iii), the function $Z: M \rightarrow \mathbb{C}$ depends just on the coordinate $r$ and, for $r \geq 0$, the absolute value $|Z(r)|$ is bounded from above by the mass of the black hole ${ }^{2}$

$$
m \geq|Z(r)|
$$

The black holes for which the inequality (4.6) is "saturated" (i.e. such that $m=\left|Z\left(r_{o}\right)\right|=$ : $\left|Z_{o}\right|$ for some $\left.r_{o} \geq 0\right)$ are called $B P S .^{3}$

On the other hand, by the Bekenstein-Hawking entropy-area formula ([7, 28]), the thermodynamical entropy $S$ of a black hole is completely determined by the area $A_{H}$ of the (unique and time independent) event horizon located at $r=0$. More precisely, in natural units

$$
S=\frac{A_{H}}{4} .
$$

This relation, together with eq. (4.3) and with the fact that, for an extremal BPS black hole (see for instance [5, 21])

$$
C^{2}=\left|Z_{o}\right|^{2}
$$

implies that for such a black hole the following relation between the entropy and the central charge holds:

$$
S=\pi\left|Z_{o}\right|^{2}
$$

The second important property of the central charge that we need to recall is a crucial phenomenon of the BPS black holes. Consider one such black hole, with magnetic and electric charges $p^{I}, q_{J}$ and mass $m \neq 0$. We recall that the scalar fields associated with such a (static and spherically symmetric) black hole are given by a map, depending only on the radius $r$,

$$
z=z(r): M \rightarrow \mathcal{S}_{\mathcal{T}}=\mathbb{R}^{n}+i \mathcal{V}=\mathbb{R}^{n}+i \mathbb{R}_{+} \cdot \mathcal{T}
$$

\footnotetext{
${ }^{2}$ By mass of a black hole we mean its Bertotti-Robinson mass [8, 36].

${ }^{3}$ The acronym BPS stands for Bogomol'nyı̆, Prasad and Sommerfield, who were the first that determined explicit solutions of supersymmetric equations, where an inequality of the form (4.6) appeared as saturated [10, 35].
} 
into the projective scalar manifold $\mathcal{S}_{\mathcal{T}}=\mathcal{M} / \mathbb{C}^{*}$, determined from a special cubic cone of $\mathcal{T} \subset\{d=1\} \subset \mathbb{R}^{n}$ through the $r$-map, which is the projectivisation of a corresponding conical scalar manifold $\mathcal{M} \subset \mathbb{C}^{n+1}$. The map $z=z(r)$ is canonically associated with the map into the conical scalar manifold $\mathcal{M}$

$$
X=X(r): M \rightarrow \mathcal{M} \subset \mathbb{C}^{n+1}, \quad X(r):=\left(1, z^{1}(r), \ldots, z^{n}(r)\right) .
$$

We also recall that the potential of the Kähler metric $g_{\mathcal{S}_{\mathcal{T}}}$ of the scalar manifold $\mathcal{S}_{\mathcal{T}}$ is the function

$$
\mathcal{K}(z, \bar{z})=-\log (8 d(\operatorname{Im}(z))
$$

and that the prepotential $F$, which characterises the conical scalar manifold $\mathcal{M} \subset \mathbb{C}^{n+1}$, is the holomorphic function

$$
F(X)=\frac{d\left(X^{1}, X^{2}, \ldots X^{n}\right)}{X^{0}}=\frac{d_{a b c} X^{a} X^{b} X^{c}}{X^{0}} .
$$

Let now $r_{o} \geq 0$ be the smallest radius such that $m=\left|Z_{o}\right|$ and denote by

$$
Z_{o}:=Z\left(r_{o}\right) \in \mathbb{C}, \quad z_{o}:=z\left(r_{o}\right) \in \mathcal{S}, \quad X_{o}=\left(1, z^{1}\left(r_{o}\right), \ldots, z^{n}\left(r_{o}\right)\right) \in \mathcal{M}
$$

the values at $r_{o}$ of the central charge $Z(r)$, of the scalar fields map $z(r)$ and of the canonical associated lifted map $X(r)=(1, z(r))$, respectively. The following crucial properties hold:

(a) $r_{o}=0$, implying that $\left|Z_{o}\right|:=\lim _{r \rightarrow 0^{+}}|Z(r)|$;

(b) denoting $F_{I}:=\frac{\partial F}{\partial X^{I}}$

$$
\begin{aligned}
& p^{K}=-e^{\frac{\mathcal{K}(z, \bar{z})}{2}} 2 \operatorname{Im}\left(\bar{Z}_{o} X_{o}^{K}\right)=-\frac{2 \operatorname{Im}\left(\bar{Z}_{o} X_{o}^{K}\right)}{\sqrt{8 d(\operatorname{Im}(z))}}, \\
& q_{L}=-e^{\frac{\mathcal{X}(z, \bar{z})}{2}} 2 \operatorname{Im}\left(\left.\bar{Z}_{o} F_{L}\right|_{\left(X_{o}^{J}\right)}\right)=-\frac{2 \operatorname{Im}\left(\left.\bar{Z}_{o} F_{L}\right|_{\left(X_{o}^{J}\right)}\right)}{\sqrt{8 d(\operatorname{Im}(z))}} .
\end{aligned}
$$

The equalities (4.10) completely determine the magnetic and electric charges in terms of the scalar fields and the central charge of the BPS black hole and they are consequences of the celebrated theory of the attractor mechanism [22, 25]. We call them BPS relations.

We derive our main results from (4.9) and (4.10). In fact, in the next section, we will show that the problem of inverting the relations (4.10) boils down to determining the inverse map to the quadratic map

$$
y \mapsto h(y)=\left(h_{a}(y):=d_{a b c} y^{b} y^{c}\right) .
$$

On the other hand, in theorem 2.11 we determined the explicit expression of such inverse map in case $d$ is an invariant cubic polynomial. Combining these results, we are able to obtain the explicit expression for the modulus $\left|Z_{o}\right|$ of the central charge of a BPS black hole - and thus, due to (4.9), for its entropy $S$ - in terms of its electric and magnetic charges only, provided that the scalar manifold $\mathcal{S}=\mathbb{R}^{n}+i \mathcal{V}$ is homogeneous. The formula we determine is valid for any choice of the homogeneous scalar manifold and extends the previously known expression, determined only for the cases in which $\mathcal{S}_{\mathcal{T}}$ is a symmetric manifold. 


\section{Recovering the central charge and the scalar fields from the electric and magnetic charges of a BPS black hole}

\subsection{The BPS relations as a map}

In this section, we discuss some aspects of the map, which determines the BPS relations between central charge and scalar fields on one side and the magnetic and electric charges on the other side. In order to make fully clear that here and in the next two subsections we address purely mathematical properties of this map - whose arguments and values are tuples of just (real or complex) numbers, not quantities with a prescribed physical meaning — we adopt the following notational conventions.

- An index running from 0 to $n$ (resp. from 1 to $n$ ) is always denoted by a capital letter as $I, J, K$, etc. (resp. small letter as $a, b, c$, etc.).

- When no ambiguity may occur, $n$-tuples as $\left(z^{a}\right),\left(\mathfrak{p}^{a}\right)$, etc., are denoted with no index, i.e. by $z, \mathfrak{p}$, etc.

- The standard pairing between a 1-form $w=\left(w_{a}\right) \in \mathbb{R}^{n \prime}:=\operatorname{Hom}\left(\mathbb{R}^{n}, \mathbb{R}\right)$ and a vector $y=\left(y^{a}\right) \in \mathbb{R}^{n}$ is denoted by $\langle w, y\rangle:=w_{a} y^{a}$.

- The standard complex coordinates of $\mathbb{C}^{n}$ and the standard real coordinates of $\mathbb{R}^{2 n+2}$ are indicated by $z=\left(z^{a}\right)$ and by $\left(\mathfrak{p}^{0}, \mathfrak{p}^{a}, \mathfrak{q}_{0}, \mathfrak{q}_{a}\right)=\left(\mathfrak{p}^{0}, \mathfrak{p}, \mathfrak{q}_{0}, \mathfrak{q}\right)$, respectively.

- We denote by $h(y)=\left(h_{a}(y):=d_{a b c} y^{b} y^{c}\right)$ the quadratic map determined by the cubic polynomial $d(y)=d_{a b c} y^{a} y^{b} y^{c}$ of the scalar manifold $\mathcal{S}_{\mathcal{T}}=\mathbb{R}^{n}+i \mathcal{V}$. We use the same symbols for their natural extensions $d: \mathbb{C}^{n} \rightarrow \mathbb{C}$ and $h: \mathbb{C}^{n} \rightarrow \mathbb{C}^{n \prime}$, defined by $d(z):=d_{a b c} z^{a} z^{b} z^{c}$ and $\left(h_{a}(z)\right):=\left(d_{a b c} z^{b} z^{c}\right)$, respectively.

From now on, we focus on the BPS map given in (1.6), namely the map

$$
\begin{aligned}
\mathfrak{f}: \mathbb{C}^{*} \times\left(\mathbb{R}^{n}+i \mathcal{V}\right) \subset \mathbb{C}^{n+1} & \longrightarrow \mathbb{R}^{2 n+2}, \\
\mathfrak{f}\left(Z, z^{a}, \bar{Z}, \bar{z}^{a}\right) & =\left(\mathfrak{p}^{K}\left(Z, z^{a}, \bar{Z}, \bar{z}^{a}\right), \mathfrak{q}_{L}\left(Z, z^{a}, \bar{Z}, \bar{z}^{a}\right)\right),
\end{aligned}
$$

defined by

$$
\begin{aligned}
& \mathfrak{p}^{0}(Z, z, \bar{Z}, \bar{z}):=\frac{\operatorname{Im} Z}{\sqrt{2 d(y)}} \\
& \mathfrak{p}^{a}(Z, z, \bar{Z}, \bar{z}):=-\frac{\operatorname{Im}\left(\bar{Z} z^{a}\right)}{\sqrt{2 d(y)}}, \\
& \mathfrak{q}_{0}(Z, z, \bar{Z}, \bar{z}):=-\frac{\operatorname{Im}\left(\bar{Z} d_{a b c} z^{a} z^{b} z^{c}\right)}{\sqrt{2 d(y)}} \\
& \mathfrak{q}_{a}(Z, z, \bar{Z}, \bar{z}):=-\frac{3 \operatorname{Im}\left(\bar{Z} d_{a b c} z^{b} z^{c}\right)}{\sqrt{2 d(y)}}, \quad \text { where } y:=\operatorname{Im}(z)
\end{aligned}
$$

The BPS relations (4.10) tell that the value $\mathfrak{f}\left(Z_{o}, z_{o}^{1}, \ldots, z_{o}^{n}\right)$ of $\mathfrak{f}$ on the central charge and the scalar fields map at the horizon $r_{o}=0$ of a BPS black hole gives the magnetic and 
electric charges of the black hole

$$
\left(p^{K}, q_{L}\right)=\left(\mathfrak{p}^{K}\left(Z_{o}, z_{o}, \bar{Z}_{o}, \bar{z}_{o}\right), \mathfrak{q}_{L}\left(Z_{o}, z_{o}, \bar{Z}_{o}, \bar{z}_{o}\right)\right) .
$$

In what follows, we decompose the standard coordinate of $\mathbb{C}^{*}$ as $Z=t e^{i \vartheta}$, with $t=|Z|>0$ and $\vartheta \in \mathbb{R} \bmod 2 \pi$, and we use the short-hand notation $\mathfrak{c}:=\frac{1}{\sqrt{2 d(y)}}$. In this way, using the above notational convention, the (5.2) take the form

$$
\begin{aligned}
\mathfrak{p}^{0} & =\mathfrak{c} t \sin \vartheta, \\
\mathfrak{p} & =-\mathfrak{c} t \operatorname{Im}\left(e^{-i \vartheta} z\right), \\
\mathfrak{q}_{0} & =\mathfrak{c} t \operatorname{Im}\left(e^{-i \vartheta}(d(z))\right), \\
\mathfrak{q} & =-3 \mathfrak{c t} \operatorname{Im}\left(e^{-i \vartheta} h(z)\right) .
\end{aligned}
$$

We now consider the purely mathematical questions of finding the domains $\mathcal{U} \subset \mathbb{C}^{*} \times \mathcal{S}$ on which the BPS map $\mathfrak{f}$ is locally invertible and, on such domains, determining the explicit expressions for the (local) inverses of this map. For this purpose, it is convenient to split the domain $\mathbb{C}^{*} \times \mathcal{S}$ of the BPS map into the union of the following four disjoint subsets:

- the $(2 n+1)$-dimensional hypersurfaces

$$
\mathcal{C}_{+}:=\{\vartheta=0\} \times \mathcal{S}, \quad \mathcal{C}_{-}:=\{\vartheta=\pi\} \times \mathcal{S},
$$

- the $(2 n+2)$-dimensional open subsets

$$
\mathcal{A}_{+}:=\{\vartheta \in(0, \pi)\} \times \mathcal{S}, \quad \mathcal{A}_{-}:=\{\vartheta \in(-\pi, 0)\} \times \mathcal{S} .
$$

We discuss the behaviour of the restrictions $\left.\mathfrak{f}\right|_{\mathcal{C}_{ \pm}}$and $\left.\mathfrak{f}\right|_{\mathcal{A}_{ \pm}}$separately.

\subsection{The maps $\left.\mathfrak{f}\right|_{\mathcal{C}_{ \pm}}$take values into $\left\{\mathfrak{p}^{0}=0\right\}$ and are globally invertible}

Due to (5.3), the map $\mathfrak{f}$ sends both hypersurfaces $\mathcal{C}_{ \pm}$into the hypersurface $\left\{\mathfrak{p}^{0}=0\right\}$ of $\mathbb{R}^{2 n+2}$. Moreover we have the following

Proposition 5.1. Each map

$$
\left.\mathfrak{f}\right|_{\mathcal{C}_{ \pm}}: \mathcal{C}_{ \pm} \rightarrow \mathfrak{f}\left(\mathcal{C}_{ \pm}\right) \subset\left\{\mathfrak{p}^{0}=0\right\}
$$

is a diffeomorphism onto its image. More precisely, if $\left(\mathfrak{p}^{0}=0, \mathfrak{p}, \mathfrak{q}_{0}, \mathfrak{p}\right)$ is a point of $\mathfrak{f}\left(\mathcal{C}_{ \pm}\right)$, then there is a unique $(t, z) \in \mathcal{C}_{ \pm}$such that $\left(\mathfrak{p}^{0}=0, \mathfrak{p}, \mathfrak{q}_{0}, \mathfrak{p}\right)=\mathfrak{f}(t, z)$. This point is given by ${ }^{4}$

$$
\begin{aligned}
& z=\frac{1}{6} \mathcal{D}(\mathfrak{p}, \mathfrak{q})-i \mathfrak{p} \sqrt{\frac{\langle\mathfrak{q}, \mathcal{D}(\mathfrak{p}, \mathfrak{q})\rangle+12 \mathfrak{q}_{0}}{12 d(\mathfrak{p})},} \\
& t=\left(\frac{d(\mathfrak{p})\left(\langle\mathfrak{q}, \mathcal{D}(\mathfrak{p}, \mathfrak{q})\rangle+12 \mathfrak{q}_{0}\right)}{12}\right)^{\frac{1}{4}},
\end{aligned}
$$

where $\mathcal{D}^{a}(\mathfrak{p}, \mathfrak{q}):=\mathcal{D}^{a b}(\mathfrak{p}) \mathfrak{q}_{b}$ with $\left(\mathcal{D}^{a b}(\mathfrak{p})\right)$ inverse matrix of $\left(d_{a b c} \mathfrak{p}^{c}\right)$.

\footnotetext{
${ }^{4}$ Note that (5.8) gives the well known expression $S=\pi \sqrt{\frac{1}{3} d(\mathfrak{p})\left(\langle\mathfrak{q}, \mathcal{D}\rangle+12 \mathfrak{q}_{0}\right)}$ for the entropy of a BPS black hole with $\mathfrak{p}^{0}=0$ due to Shmakova [38]. We also remark that, from (5.7) and the fact that $d(\mathfrak{p})<0$, we also have that $z=\frac{1}{6} \mathcal{D}(\mathfrak{p}, \mathfrak{q})+i \frac{\mathfrak{p}}{2} \frac{S}{\pi} \frac{1}{d(\mathfrak{p})}$. This amends an error in [38, formula (27)].
} 
Proof. We give the proof only for $\left.\mathfrak{f}\right|_{\mathcal{C}_{+}}$, the other being similar. Given $\left(\mathfrak{p}^{0}=0, \mathfrak{p}, \mathfrak{q}_{0}, \mathfrak{p}\right) \in$ $\mathfrak{f}\left(\mathcal{C}_{+}\right)$, any pre-image $(t, z=x+i y) \in \mathcal{C}_{+}$satisfies

$$
\begin{aligned}
\mathfrak{p} & =-\mathfrak{c} t y \quad \Longrightarrow \quad y=-\frac{\mathfrak{p}}{\mathfrak{c} t} \\
\mathfrak{q}_{0} & =3\langle h(x), \mathfrak{c} t y\rangle-\frac{1}{\mathfrak{c}^{2} t^{2}} d(\mathfrak{c} t y) \\
\mathfrak{q} & =-6 d \cdot b c x^{b}\left(\mathfrak{c} t y^{c}\right), \quad \text { with } \quad \mathfrak{c}:=\frac{1}{\sqrt{2 d(y)}} .
\end{aligned}
$$

Replacing (5.9) into (5.10) and (5.11), we get

$$
\mathfrak{c} t=\sqrt{\frac{d(\mathfrak{p})}{3\langle h(x), \mathfrak{p}\rangle+\mathfrak{q}_{0}}}, \quad \mathfrak{q}=6 d_{. b c} x^{b_{\mathfrak{p}}} .
$$

It follows that

$$
x=\frac{1}{6} \mathcal{D}^{\cdot b}(\mathfrak{p}) \mathfrak{q}_{b}, \quad \mathfrak{c} t=\sqrt{\frac{12 d(\mathfrak{p})}{\left\langle\mathfrak{q}, \mathcal{D}^{\cdot b}(\mathfrak{p}) \mathfrak{q}_{b}\right\rangle+12 \mathfrak{q}_{0}}}, \quad y=-\frac{\mathfrak{p}}{\mathfrak{c} t}=-\mathfrak{p} \sqrt{\frac{\left\langle\mathfrak{q}, \mathcal{D}^{b}(\mathfrak{p}) \mathfrak{q}_{b}\right\rangle+12 \mathfrak{q}_{0}}{12 d(\mathfrak{p})}},
$$

and $\mathfrak{c}^{2}=\frac{1}{2 d(y)}=12 \sqrt{\frac{3 d(\mathfrak{p})}{\left(\left\langle\mathfrak{q}, \mathcal{D}^{\cdot b}\left(\mathfrak{p}_{0}\right) \mathfrak{q}_{b}\right\rangle+12 \mathfrak{q}_{0}\right)^{3}}}$. This and (5.13) imply the claim.

\subsection{The maps $\left.\mathfrak{f}\right|_{\mathcal{A}_{ \pm}}$take values into $\left\{\mathfrak{p}^{0} \neq 0\right\}$ and are locally invertible}

The situation for the restriction of $\mathfrak{f}$ on the sets $\mathcal{A}_{ \pm} \subset \mathcal{S} \times \mathbb{C}^{*} \backslash\left(\mathcal{C}_{+} \cup \mathcal{C}_{-}\right)$is quite different from the previous. First of all, by (5.3), it is clear that the images $\mathfrak{f}\left(\mathcal{A}_{ \pm}\right)$are included in the open subset $\left\{\mathfrak{p}^{0} \neq 0\right\} \subset \mathbb{R}^{2 n+2}$. Moreover

Theorem 5.2. Each map $\left.\mathfrak{f}\right|_{\mathcal{A}_{ \pm}}: \mathcal{A}_{ \pm} \rightarrow \mathfrak{f}\left(\mathcal{A}_{ \pm}\right) \subset\left\{\mathfrak{p}^{0} \neq 0\right\}$ is a local diffeomorphism and the (locally defined) inverse map

$$
(\mathfrak{p}, \mathfrak{q}) \rightarrow\left(t e^{\vartheta}, z\right)
$$

is given by

$$
\begin{aligned}
& z:=\frac{e^{i \vartheta}}{\mathfrak{p}^{0}} h^{-1}\left(h(\mathfrak{p})-\frac{1}{3} \mathfrak{p}^{0} \mathfrak{q}\right)+\frac{\mathfrak{p}}{\mathfrak{p}^{0}} \\
& \vartheta:= \pm \arccos \left(\frac{\mathfrak{p}^{0}\left(\mathfrak{p}^{0} \mathfrak{q} 0+\langle\mathfrak{q}, \mathfrak{p}\rangle\right)-2 d(\mathfrak{p})}{2\left(\mathfrak{p}^{0}\right)^{3}\left\langle h(\mathfrak{p})-\frac{1}{3} \mathfrak{p}^{0} \mathfrak{q}, h^{-1}\left(h(\mathfrak{p})-\frac{1}{3} \mathfrak{p}^{0} \mathfrak{q}\right)\right\rangle}\right) \\
& t:=\sqrt{2} \sqrt{\frac{\sin \vartheta}{\mathfrak{p}^{0}}\left\langle h(\mathfrak{p})-\frac{1}{3} \mathfrak{p}^{0} \mathfrak{q}, h^{-1}\left(h(\mathfrak{p})-\frac{1}{3} \mathfrak{p}^{0} \mathfrak{q}\right)\right\rangle}
\end{aligned}
$$

More precisely, given $\left(t_{o} e^{i \vartheta_{o}}, z_{o}\right) \in \mathcal{A}_{ \pm}$and its image $\left(\mathfrak{p}_{o}^{I}, \mathfrak{q}_{o J}\right)=\mathfrak{f}\left(t_{o} e^{i \vartheta_{o}}, z_{o}\right)$, there exist two neighbourhoods $\mathcal{U}, \mathfrak{U}^{\prime}$ of $\left(t_{o} e^{i \vartheta_{o}}, z_{o}\right)$ and $\left(\mathfrak{p}_{o}^{I}, \mathfrak{q}_{o J}\right)$, respectively, such that the restricted map $\mathfrak{f} \mid \mathfrak{u}$ is a diffeomorphism from $\mathcal{U}$ into $\mathcal{U}^{\prime}$ and its inverse $\mathfrak{F}=(\mathfrak{f} \mid \mathfrak{u})^{-1}: \mathcal{U}^{\prime} \rightarrow \mathcal{U}$ is given by (5.14)-(5.16). 
The neighbourhood $\mathcal{U}^{\prime} \subset \mathbb{R}^{2 n+2}$ of $\left(\mathfrak{p}_{o}^{I}, \mathfrak{q}_{o J}\right)$ is defined as follows. Consider the vector $v_{o}$ and the 1-form $\alpha_{o}$ defined by

$$
v_{o}:=\frac{t_{o} y_{o}}{\sqrt{2 d\left(y_{o}\right)}} \in \mathcal{V}_{d} \subset \mathbb{R}^{n}, \quad \alpha_{o}:=3 h\left(\mathfrak{p}_{o}\right)-\mathfrak{p}_{o}^{0} \mathfrak{q}_{o} \in \mathbb{R}^{n *}
$$

Then $\alpha_{o}$ belongs to the cone $h\left(\mathcal{V}_{d}\right) \subset h(\{d>0\})$ and there are two neighbourhoods $\mathcal{W}_{1} \subset \mathbb{R}^{n}$ and $\mathcal{W}_{2}^{*} \subset \mathbb{R}^{n *}$ of $v_{o}$ and $\alpha_{o}$, respectively, such that the restriction $\left.h\right|_{\mathcal{W}_{1}}: \mathcal{W}_{1} \rightarrow \mathcal{W}_{2}^{*}$ is a diffeomorphism between such two neighbourhoods. Then $\mathrm{U}^{\prime}$ is the open set

$$
\mathcal{U}^{\prime}:=\left\{\left(\mathfrak{p}^{I}, \mathfrak{q}_{J}\right): \mathfrak{p}^{0} \neq 0, \quad 3 h(\mathfrak{p})-\mathfrak{p}^{0} \mathfrak{q} \in \mathcal{W}_{2}^{*}, \quad \operatorname{sign}\left(\mathfrak{p}^{0}\right)=\operatorname{sign}\left(\mathfrak{p}_{o}^{0}\right)\right\} .
$$

The corresponding neighbourhood $\mathcal{U} \subset \mathbb{C}^{n+1}$ of $\left(t_{o} e^{i \vartheta_{o}}, z_{o}\right)$ is given by the images of $\mathfrak{U}^{\prime}$ under the map (5.14)-(5.16).

Proof. (1) Consider the change of coordinates on $\{\operatorname{Im}(z) \neq 0\} \times \mathbb{C}^{n} \subset \mathbb{C}^{n+1}$

$$
\left(t e^{i \vartheta}, z^{a}\right) \longmapsto\left(t e^{i \vartheta}, \widetilde{z}^{a}=e^{-i \vartheta} z^{a}\right) .
$$

In these new coordinates, the components (5.4) and (5.6) of $\mathfrak{f}$ become (here, $\widetilde{x}:=\operatorname{Re}(\widetilde{z})$ and $\widetilde{y}:=\operatorname{Im}(\widetilde{z}))$

$$
\mathfrak{p}=-\mathfrak{c} t \widetilde{y}, \quad \mathfrak{q}=3 \mathfrak{c} t \operatorname{Re}\left(i e^{i \vartheta} h(\widetilde{z})\right) .
$$

Using the first of these two relations, we may rewrite the second as

$$
\begin{array}{r}
\mathfrak{q}=3 \mathfrak{c t} \operatorname{Re}\left((i \cos \vartheta-\sin \vartheta)\left(h(\widetilde{x})-h(\widetilde{y})+2 i d_{(\cdot) b c} \widetilde{x}^{b} \widetilde{y}^{c}\right)\right)= \\
=3 \mathfrak{c t}\left(-\sin \vartheta h(\widetilde{x})-2 \cos \vartheta d_{(\cdot) b c} \widetilde{x}^{b} \widetilde{y}^{c}\right)+3 \mathfrak{c t} \sin \vartheta h(\widetilde{y})= \\
=3 \mathfrak{c t} \sin \vartheta\left(-h(\widetilde{x})-2 \frac{\cos \vartheta}{\sin \vartheta} d_{(\cdot) b c} \widetilde{x}^{b} \widetilde{y}^{c}-\frac{\cos ^{2} \vartheta}{\sin ^{2} \vartheta} h(\widetilde{y})\right)+3 \mathfrak{c t} \frac{\sin ^{2} \vartheta+\cos ^{2} \vartheta}{\sin \vartheta} h(\widetilde{y})= \\
=-3 \mathfrak{c t} \sin \vartheta h\left(\widetilde{x}+\frac{\cos \vartheta}{\sin \vartheta} \widetilde{y}\right)+\frac{3}{\mathfrak{c t} \sin \vartheta} h(\mathfrak{p}) .
\end{array}
$$

On the other hand, from (5.3) we may replace $\mathfrak{p}^{0}=-\mathfrak{c} t \sin \vartheta$ at all points. Since $y=$ $\operatorname{Im}\left(e^{i \vartheta} \widetilde{z}\right)=\sin \vartheta \widetilde{x}+\cos \vartheta \widetilde{y}$, we have that (5.19) implies

$$
\begin{aligned}
\frac{3 \mathfrak{c}^{2} t^{2}}{\mathfrak{p}^{0}} h(y) & =\frac{3 \mathfrak{c} t}{\sin \vartheta} h\left(\sin \vartheta\left(\widetilde{x}+\frac{\cos \vartheta}{\sin \vartheta} \widetilde{y}\right)\right)= \\
& =3 \mathfrak{c t} \sin \vartheta h\left(\widetilde{x}+\frac{\cos \vartheta}{\sin \vartheta} \widetilde{y}\right)=\frac{3}{\mathfrak{p}_{0}} h(\mathfrak{p})-\mathfrak{q} .
\end{aligned}
$$

This means that, if $\left(\mathfrak{p}_{o}^{I}, \mathfrak{q}_{o J}\right)=\mathfrak{f}\left(t_{o} e^{i \vartheta_{o}}, z_{o}\right)$ for some $\left.\left(t_{o} e^{i \vartheta_{o}}, z_{o}\right) \in\{\operatorname{Im} Z \neq 0\}\right) \times \mathcal{S}$, the 1 -form $\alpha_{o}:=3 h\left(\mathfrak{p}_{o}\right)-\mathfrak{q}_{o} \mathfrak{p}_{o}^{0} \in \mathbb{R}^{n *}$ is actually equal to

$$
\alpha_{o}=3 \mathfrak{c}_{o}^{2} t_{o}^{2} h\left(y_{o}\right)=h\left(v_{o}\right), \quad v_{o}:=\sqrt{3} \mathfrak{c}_{o} t_{o} y_{o}
$$

that is $\alpha_{o}$ is the image of the element $v_{o}$ of the cone $\nu_{d}$ under the map $h$. We now observe that, at each point $y \in \mathcal{V}_{d}$, the Jacobian of the map $h$ is $\left.J h\right|_{y}=2\left(d_{a b c} y^{c}\right)$. Being 
$d$ associated with a special cubic, this matrix is non-degenerate. Hence, by the Inverse Function Theorem, there exists a neighbourhood $\mathcal{W}_{1}$ of $v_{o} \in \mathcal{V}_{d}$ and neighbourhood $\mathcal{W}_{2}^{*}$ of $\alpha_{o}$ such the restriction $-\left.h\right|_{\mathcal{W}_{1}}: \mathcal{W}_{1} \rightarrow \mathcal{W}_{2}^{*}$ admits an inverse $h^{-1}: \mathcal{W}_{2}^{*} \rightarrow \mathcal{W}_{1}$.

We now claim that if $\left(\mathfrak{p}^{I}, \mathfrak{q}_{J}\right)$ is in the open subset defined in (5.17), then there exists at least one point $\left(t e^{i \vartheta}, z=x+i y\right) \in\{\operatorname{Im}(Z) \neq 0\} \times \mathcal{S}$ which is mapped onto $\left(\mathfrak{p}^{I}, \mathfrak{q}_{J}\right)$ by $\mathfrak{f}$. Indeed, by (5.3), (5.18) and (5.20), if $\left(t e^{i \vartheta}, z=x+i y\right)$ is mapped onto $\left(\mathfrak{p}^{I}, \mathfrak{q}_{J}\right)$, then

$$
\begin{aligned}
t & =\frac{\mathfrak{p}^{0}}{\mathfrak{c} \sin \vartheta}, \quad \widetilde{y}=-\frac{\mathfrak{p}}{\mathfrak{c} t}=-\frac{\mathfrak{p}}{\mathfrak{p}^{0}} \sin \vartheta, \\
3\left(\mathfrak{p}^{0}\right)^{2} h\left(\widetilde{x}+\frac{\cos \vartheta}{\sin \vartheta} \widetilde{y}\right) & =3 h(\mathfrak{p})-\mathfrak{p}^{0} \mathfrak{q} .
\end{aligned}
$$

Using (5.21) and being $h$ is quadratic, (5.22) can be transformed into

$$
h\left(\mathfrak{p}^{0} \widetilde{x}-\cos \vartheta \mathfrak{p}\right)=h(\mathfrak{p})-\frac{1}{3} \mathfrak{p}^{0} \mathfrak{q} .
$$

Using the inverse map $h^{-1}: \mathcal{W}_{2}^{*} \rightarrow \mathcal{W}_{1}$, this condition becomes equivalent to

$$
\widetilde{x}=\frac{1}{\mathfrak{p}^{0}} h^{-1}\left(h(\mathfrak{p})-\frac{1}{3} \mathfrak{p}^{0} \mathfrak{q}\right)+\cos \vartheta \frac{\mathfrak{p}}{\mathfrak{p}^{0}} .
$$

Since $z=x+i y=e^{i \vartheta}(\widetilde{x}+i \widetilde{y})$, from (5.21) and (5.23) we get

$$
z=e^{i \vartheta} A+\frac{\mathfrak{p}}{\mathfrak{p}^{0}} \quad \text { with } \quad A:=\frac{1}{\mathfrak{p}^{0}} h^{-1}\left(h(\mathfrak{p})-\frac{1}{3} \mathfrak{p}^{0} \mathfrak{q}\right) .
$$

Plugging this and $t=\frac{\mathfrak{p}^{0}}{\mathfrak{c} \sin \vartheta}$ into $(5.5)$ and using $h \circ h^{-1}=\left.\operatorname{Id}\right|_{\mathcal{W}_{2}^{*}}$, we obtain

$$
\begin{gathered}
\mathfrak{q}_{0}=-\mathfrak{c} t \operatorname{Re}\left(i e^{-i \vartheta} d\left(e^{i \vartheta} A+\frac{\mathfrak{p}}{\mathfrak{p}^{0}}\right)\right)= \\
=-\mathfrak{c t} \operatorname{Re}\left(i e^{2 i \vartheta} d(A)\right)-\frac{\mathfrak{c} t}{\left(\mathfrak{p}^{0}\right)^{3}} \operatorname{Re}\left(i e^{-i \vartheta} d(p)\right)-3 \frac{\mathfrak{c} t}{\mathfrak{p}^{0}} \operatorname{Re}\left(i e^{i \vartheta}\langle h(A), \mathfrak{p}\rangle\right)= \\
=2 \mathfrak{c} t \sin \vartheta \cos \vartheta d(A)-\frac{\mathfrak{c} t \sin \vartheta}{\left(\mathfrak{p}^{0}\right)^{3}} d(p)+3 \frac{\mathfrak{c} t \sin \vartheta}{\mathfrak{p}^{0}}\langle h(A), \mathfrak{p}\rangle= \\
=2 \cos \vartheta \mathfrak{p}^{0} d(A)-\frac{d(p)}{\left(\mathfrak{p}^{0}\right)^{2}}+\frac{3}{\left(\mathfrak{p}^{0}\right)^{2}}\left\langle h(\mathfrak{p})-\frac{1}{3} \mathfrak{p}^{0} \mathfrak{q}, \mathfrak{p}\right\rangle= \\
=2 \cos \vartheta \mathfrak{p}^{0} d(A)+2 \frac{d(p)}{\left(\mathfrak{p}^{0}\right)^{2}}-\frac{1}{\mathfrak{p}^{0}}\langle\mathfrak{q}, \mathfrak{p}\rangle .
\end{gathered}
$$

Solving this equation with respect to $\cos \vartheta$ we obtain

$$
\cos \vartheta=\frac{\mathfrak{q}^{0}}{2 \mathfrak{p}^{0} d(A)}-\frac{d(p)}{\left(\mathfrak{p}^{0}\right)^{3} d(A)}+\frac{1}{2\left(\mathfrak{p}^{0}\right)^{2} d(A)}\langle\mathfrak{q}, \mathfrak{p}\rangle,
$$

and hence

$$
\sin \vartheta= \pm \sqrt{1-\cos ^{2} \vartheta}= \pm \sqrt{1-\left(\frac{\mathfrak{q}^{0}}{2 \mathfrak{p}^{0} d(A)}-\frac{d(\mathfrak{p})}{\left(\mathfrak{p}^{0}\right)^{3} d(A)}+\frac{1}{2\left(\mathfrak{p}^{0}\right)^{2} d(A)}\langle\mathfrak{q}, \mathfrak{p}\rangle\right)^{2}}
$$


the sign being equal to +1 in case $\vartheta \in(0, \pi)$ (that is, in case $\mathfrak{p}^{0}>0$ ) and equal to -1 otherwise. In both cases $\frac{\sin \vartheta}{\mathfrak{p}^{0}}>0$. In order to conclude, we only need to recall that

$$
\begin{aligned}
t=\sqrt{\frac{\left(\mathfrak{p}^{0}\right)^{2}}{\mathfrak{c}^{2} \sin ^{2} \vartheta}=} & \sqrt{\frac{2\left(\mathfrak{p}^{0}\right)^{2} d(y)}{\sin ^{2} \vartheta}}=\sqrt{2\left(\mathfrak{p}^{0}\right)^{2} \sin \vartheta d(A)}= \\
=\sqrt{2 \frac{\sin \vartheta}{\mathfrak{p}^{0}} d\left(\mathfrak{p}^{0} A\right)} & =\sqrt{2 \frac{\sin \vartheta}{\mathfrak{p}^{0}} d\left(h^{-1}\left(h(\mathfrak{p})-\frac{1}{3} \mathfrak{p}^{0} \mathfrak{q}\right)\right)}= \\
& =\sqrt{2} \sqrt{\frac{\sin \vartheta}{\mathfrak{p}^{0}}\left\langle h(\mathfrak{p})-\frac{1}{3} \mathfrak{p}^{0} \mathfrak{q}, h^{-1}\left(h(\mathfrak{p})-\frac{1}{3} \mathfrak{p}^{0} \mathfrak{q}\right)\right\rangle} .
\end{aligned}
$$

Finally, we observe that, since $\frac{\sin \vartheta}{\mathfrak{p}^{0}}>0$, the equality (5.28) makes sense only if

$$
\left\langle h(\mathfrak{p})-\frac{1}{3} \mathfrak{p}^{0} \mathfrak{q}, h^{-1}\left(h(\mathfrak{p})-\frac{1}{3} \mathfrak{p}^{0} \mathfrak{q}\right)\right\rangle>0 .
$$

Remark 5.3. A few simple algebraic manipulations of the (5.14)-(5.16) lead to the same formulas for the absolute value of the central charge (hence, also for the entropy $S$ ) and for the scalar fields at the horizon of a BPS black hole obtained in [38] by a different line of arguments. Indeed, given a point $\left(\mathfrak{p}^{I}, \mathfrak{q}_{J}\right)=\mathfrak{f}\left(t e^{i \vartheta}, z\right) \in \mathfrak{f}\left(\mathcal{A}_{ \pm}\right)$, let $\mathcal{X}$ and $\Delta=\left(\Delta_{a}\right)$ Shmakova's vector and 1 -form defined by

$$
\mathcal{X}:=\sqrt{3} h^{-1}\left(h(\mathfrak{p})-\frac{1}{3} \mathfrak{p}^{0} \mathfrak{q}\right)=\sqrt{3} \mathfrak{p}^{0} A, \quad \Delta:=3\left(h(\mathfrak{p})-\frac{1}{3} \mathfrak{p}^{0} \mathfrak{q}\right) .
$$

Then (5.29) is equivalent to $\frac{1}{3^{\frac{3}{2}}}\langle\Delta, \mathcal{X}\rangle>0$. Using this and (5.27) we have that

$$
\begin{aligned}
& t^{2}= \pm \frac{2}{(3)^{\frac{3}{2}}} \frac{1}{\mathfrak{p}^{0}}\langle\Delta, \mathcal{X}\rangle \sqrt{1-\left(\frac{3^{\frac{3}{2}} \mathfrak{q}^{0}\left(\mathfrak{p}^{0}\right)^{2}}{2 d\left(\sqrt{3} \mathfrak{p}^{0} A\right)}-\frac{3^{\frac{3}{2}} d(\mathfrak{p})}{d\left(\sqrt{3} \mathfrak{p}^{0} A\right)}+\frac{3^{\frac{3}{2}} \mathfrak{p}^{0}}{2 d\left(\sqrt{3} \mathfrak{p}^{0} A\right)}\langle\mathfrak{q}, \mathfrak{p}\rangle\right)^{2}}= \\
& = \pm \frac{1}{3 \mathfrak{p}^{0}} \sqrt{\frac{4}{3}\langle\mathcal{X}, \Delta\rangle^{2}-9\left(\mathfrak{p}^{0}\left(\mathfrak{q}^{0} \mathfrak{p}^{0}+\langle\mathfrak{q}, \mathfrak{p}\rangle\right)-2 d(\mathfrak{p})\right)^{2}}=
\end{aligned}
$$

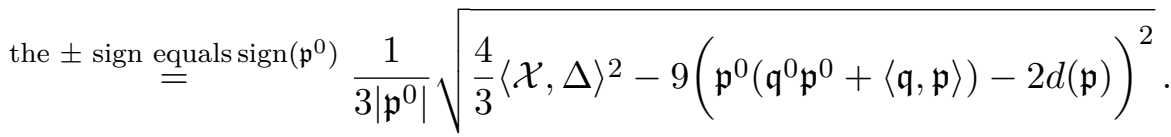

This matches [38, formula (12)]. Similar straightforward algebraic manipulations of the (5.14)-(5.16) lead to the expression

$$
z=\left(\frac{1}{\mathfrak{p}^{0}} \mathfrak{p}+\frac{3}{2} \frac{\mathfrak{p}^{0}\left(\mathfrak{p}^{0} \mathfrak{q}_{0}+\langle\mathfrak{q}, \mathfrak{p}\rangle\right)-2 d(\mathfrak{p})}{\mathfrak{p}^{0} d(\mathcal{X})} \mathcal{X}\right)+i \frac{3 t^{2}}{2 d(\mathcal{X})} \mathcal{X}
$$

which matches $[38$, formula $(24)] .^{5}$

\footnotetext{
${ }^{5}$ There is just a sign change that is due to our different assumptions. In fact, according to them, we have $d(y)>0$ - and not $d(y)<0$ - at the points $z=x+i y$ of $\mathcal{S}$.
} 
The local invertibility property established in theorem 5.2 has in practice the following meaning. Assume that $\mathfrak{p}^{I}, \mathfrak{q}_{J}$ are the values of the magnetic and electric charges of a BPS black hole and that $Z_{o}=t_{o} e^{i \vartheta_{o}}:=Z\left(r_{o}\right)$ and $z_{o}:=z\left(r_{o}\right)$ are the corresponding values of the central charge and of the scalar fields map at the horizon $r_{o}=0$. Since the result does not guarantees that $\left.\mathfrak{f}\right|_{\mathcal{A}_{ \pm}}$is globally invertible, it does not exclude the possibility that there is some choice for the cubic polynomial $d$, which allows the existence of several different BPS black holes, all of them with the same electric and magnetic charges, but also each of them with distinct horizon values for the central charge or scalar fields. In other words, for an appropriate choice of $d$, it might be that there are distinct BPS black holes with charges and horizon values for the central charge and the scalar fields with

$$
\left(p^{I}, q_{K}\right)=\left(p^{\prime I}, q_{K}^{\prime}\right)=\left(p^{\prime \prime I}, q_{K}^{\prime \prime}\right)=\ldots \text { but }\left(Z_{o}, z_{o}\right) \neq\left(Z_{o}^{\prime}, z_{o}^{\prime}\right) \neq\left(Z_{o}^{\prime \prime}, z_{o}^{\prime \prime}\right) \neq \ldots
$$

However, even if this is the case, the pairs $\left(z_{o}, Z_{o}\right) \neq\left(z_{o}^{\prime}, Z_{o}^{\prime}\right) \neq\left(z_{o}^{\prime \prime}, Z_{o}^{\prime \prime}\right) \neq \ldots$ constitute a discrete set of points in $\mathcal{A}_{+} \cup \mathcal{A}_{-} \subset \mathbb{C}^{*} \times \mathcal{S}_{\mathcal{T}}$. Indeed, for each such pair there must be a neighbourhood on which the BPS map is one-to-one.

We finally stress the fact that the proof of theorem 5.2 shows that there exists a global inverse each map $\mathfrak{f}: \mathcal{A}_{ \pm} \rightarrow \mathfrak{f}\left(\mathcal{A}_{ \pm}\right) \subset \mathbb{R}^{2 n+2}$ if and only if the restriction to $\mathcal{V}$ of $h$ admits a global inverse, namely

Corollary 5.4. If the restriction $\left.h\right|_{\mathcal{V}}: \mathcal{V} \rightarrow h(\mathcal{V}) \subset \mathbb{R}^{n \prime}$ admits an inverse $\left(\left.h\right|_{\mathcal{V}}\right)^{-1}: h(\mathcal{V}) \rightarrow$ $\mathcal{V}$, then each of the two maps $\left.\mathfrak{f}\right|_{\mathcal{A}_{ \pm}}: \mathcal{A}_{ \pm} \rightarrow \mathfrak{f}\left(\mathcal{A}_{ \pm}\right) \subset \mathbb{R}^{2 n+2}$ is a diffeomorphism onto its image, with inverse given by (5.14)-(5.16).

\subsection{The entropy of BPS black holes in case of homogeneous scalar manifolds}

By corollary 5.4, theorem 3.2, theorem 2.11, lemma 2.10, if the scalar manifold $\mathcal{S}_{\mathcal{T}}=\mathbb{R}^{n}+i \mathcal{V}$, $\mathcal{V}=\mathbb{R}_{+} \cdot \mathcal{T} \subset\{d>0\}$, is homogeneous and determined by an irreducible invariant cubic polynomial $d=k d_{v}: \mathbb{R}^{n} \rightarrow \mathbb{R}$, then $\mathcal{V}$ is a special Vinberg cone and the corresponding quadratic map $\left.h\right|_{\mathcal{V}}: \mathcal{V} \subset \mathbb{R}^{n} \rightarrow-\mathcal{V}^{\prime} \subset \mathbb{R}^{n *}$ is globally invertible with inverse given by

$$
h^{-1}(y)=\frac{1}{\sqrt{d^{\prime}(y)}} h^{\prime}(y) \quad \text { with } d^{\prime}=\frac{1}{k} d_{v}^{\prime} \text { dual invariant cubic polynomial } .
$$

As a consequence, the entropy $S=\pi|Z|^{2}$ of an extremal BPS black hole with magnetic charge $p^{0} \neq 0$ is uniquely determined by the black hole charges $\left(p^{I}, q_{J}\right)=\left(p^{0}, p, q_{0}, q\right)$ by means of the formula (5.31), which provides $t^{2}=|Z|^{2}$. More precisely, since in the homogeneous case the map $h^{-1}$ is given by (5.33), the term $\langle\Delta, \mathcal{X}\rangle$ in (5.31) can be written as

$$
\langle\Delta, \mathcal{X}\rangle=3^{\frac{3}{2}}\left\langle h(p)-\frac{1}{3} p^{0} q, h^{-1}\left(h(p)-\frac{1}{3} p^{0} q\right)\right\rangle=3^{\frac{3}{2}} \sqrt{d^{\prime}\left(h(p)-\frac{1}{3} p^{0} q\right)} .
$$

It follows that

$$
S=\pi \sqrt{I_{4}} \quad \text { with } \quad I_{4}=\frac{4}{\left(p^{0}\right)^{2}} d^{\prime}\left(h(p)-\frac{1}{3} p^{0} q\right)-\frac{1}{\left(p^{0}\right)^{2}}\left(\left(q_{0} p^{0}+\langle q, p\rangle\right) p^{0}-2 d(p)\right)^{2} .
$$


It is a remarkable fact that, this rational function is actually a quartic polynomial. Indeed, it is straightforward to check that, due to the identity (2.42),

$$
\begin{gathered}
\frac{4}{\left(p^{0}\right)^{2}} d^{\prime}\left(h(p)-\frac{1}{3} p^{0} q\right)=\frac{4}{\left(p^{0}\right)^{2}}\left\langle h^{\prime}(h(p)), h(p)\right\rangle- \\
\quad-\frac{4}{p^{0}}\left\langle h^{\prime}(h(p)), q\right\rangle+\frac{4}{3} d^{\prime}(q)-\frac{4 p^{0}}{3^{3}}\left\langle h^{\prime}(q), q\right\rangle= \\
=\frac{4}{\left(p^{0}\right)^{2}}(d(p))^{2}-\frac{4}{p^{0}} d(p)\langle p, q\rangle+\frac{4}{3}\left\langle h(p), h^{\prime}(q)\right\rangle-\frac{4 p^{0}}{3^{3}} d^{\prime}(q), \\
-\frac{1}{\left(p^{0}\right)^{2}}\left(p^{0}\left(p^{0} q_{0}+\langle q, p\rangle\right)-2 d(p)\right)^{2}= \\
=-\left(q_{0} p^{0}+\langle q, p\rangle\right)^{2}+4 q_{0} d(p)+\frac{4}{p^{0}}\langle q, p\rangle d(p)-\frac{4}{\left(p^{0}\right)^{2}}(d(p))^{2}
\end{gathered}
$$

which imply

$$
I_{4}=-\left(q_{0} p^{0}+\langle q, p\rangle\right)^{2}+4 q_{0} d(p)-\frac{4}{27} p^{0} d^{\prime}(q)+\frac{4}{3}\left\langle h(p), h^{\prime}(q)\right\rangle .
$$

Summing up, we have the following final result:

Theorem 5.5. In ungauged $N=2 D=4$ supergravity with homogeneous scalar manifold $\mathcal{S}$ (not necessarily symmetric) given by an irreducible cubic polynomial d (see section 4.1), the entropy of the extremal BPS black holes defined in section 4.3 is expressed in terms of their magnetic and electric charges $\left(p^{0}, p, q_{0}, q\right)$ by

$$
S=\pi \sqrt{-\left(q^{0} p^{0}+\langle q, p\rangle\right)^{2}+4 q_{0} d(p)-\frac{4}{27} p^{0} d^{\prime}(q)+\frac{4}{3}\left\langle h(p), h^{\prime}(q)\right\rangle} .
$$

Note that, in the cases in which $\mathcal{S}$ is symmetric, (5.37) coincides with the entropy formula which was so far known [20]. To check this, one should first recall that, in the physics literature, the prepotential is usually assumed to have the form $F(X)=\frac{1}{3 !} \frac{D_{a b c} X^{a} X^{b} X^{c}}{X^{0}}$, meaning that $D$ and our cubic polynomial $d$ are related by $d=\frac{1}{6} D$. Then, we remind that if the scalar manifold $\mathcal{S}=\mathbb{R}^{n}+i \mathcal{V}$ is symmetric, it has for long time known that there exists a symmetric contravariant cubic tensor $D^{\prime}\left(Y^{b}\right)=D^{\prime a b c} Y_{a} Y_{b} Y_{c}$ satisfying the "adjoint identity" $[15,27]$

$$
D^{\prime}(D(X), D(X))=\frac{4}{3} D(X) X
$$

By substituting $D=6 d$ and comparing with (2.42), we see that the contravariant tensor $D^{\prime}$ used in physics literature is related with our invariant dual cubic polynomial $d^{\prime}$ by

$$
d^{\prime}=\frac{9}{2} D^{\prime}
$$

Plugging all this in (5.36), the expression reduces to

$$
I_{4}=-\left(q_{0} p^{0}+\langle q, p\rangle\right)^{2}+4 q_{0} I_{3}(p)-4 p^{0} I_{3}(q)+4\left\{I_{3}(p), I_{3}(q)\right\}
$$


where

$$
I_{3}(p):=\frac{1}{3 !} D(p), \quad I_{3}(q):=\frac{1}{3 !} D^{\prime}(q),
$$

and $\{\cdot, \cdot\}$ stands for the standard Poisson bracket in $\mathbb{R}^{n} \times \mathbb{R}^{n *}$, which gives

$$
\left\{I_{3}(p), I_{3}(q)\right\}=\frac{1}{4}\left\langle D(p, p, \cdot), D^{\prime}(q, q, \cdot)\right\rangle=\frac{1}{3}\left\langle h(p), h^{\prime}(q)\right\rangle .
$$

The (5.39) is precisely the formula so far known only for the cases with symmetric scalar manifolds.

Open Access. This article is distributed under the terms of the Creative Commons Attribution License (CC-BY 4.0), which permits any use, distribution and reproduction in any medium, provided the original author(s) and source are credited.

\section{References}

[1] D.V. Alekseevsky and V. Cortés, Classification of N-(super)-extended Poincaré algebras and bilinear invariants of the Spinor representation of $\operatorname{Spin}(p, q)$, Commun. Math. Phys. 183 (1997) 477.

[2] D.V. Alekseevsky and V. Cortés, Special Vinberg Cones, Transform. Groups 26 (2021) 377.

[3] D.V. Alekseevsky, V. Cortés and T. Mohaupt, Conification of Kähler and hyper-Kähler manifolds, Commun. Math. Phys. 324 (2013) 637 [arXiv:1205.2964] [INSPIRE].

[4] L. Andrianopoli et al., $N=2$ supergravity and $N=2$ super Yang-Mills theory on general scalar manifolds: Symplectic covariance, gaugings and the momentum map, J. Geom. Phys. 23 (1997) 111 [hep-th/9605032] [INSPIRE].

[5] L. Andrianopoli, R. D'Auria, S. Ferrara and M. Trigiante, Extremal black holes in supergravity, in Lecture Notes in Physics 737, Springer, Berlin Germany (2008), pp. 661-672 [hep-th/0611345] [INSPIRE].

[6] R. Arnowitt, S. Deser and C.W. Misner, The Dynamics of General Relativity, in Gravitation: an Introduction to Current Research, Wiley, New York NY U.S.A. (1962), pp. 1997-2027.

[7] J.D. Bekenstein, Black holes and entropy, Phys. Rev. D 7 (1973) 2333 [inSPIRE].

[8] B. Bertotti, Uniform electromagnetic field in the theory of general relativity, Phys. Rev. 116 (1959) 1331 [INSPIRE].

[9] S. Bellucci, A. Marrani and R. Roychowdhury, Topics in Cubic Special Geometry, J. Math. Phys. 52 (2011) 082302 [arXiv: 1011.0705] [INSPIRE].

[10] E.B. Bogomol'nyı̆, Stability of Classical Solutions (in Russian), Yad. Fiz. 24 (1976) 861 [Sov. J. Nucl. Phys. 24 (1976) 449] (English translation) [INSPIRE].

[11] S. Cecotti, Homogeneous Kähler Manifolds and T Algebras in $N=2$ Supergravity and Superstrings, Commun. Math. Phys. 124 (1989) 23 [INSPIRE].

[12] V. Cortés, Homogeneous special geometry, Transform. Groups 1 (1996) 337.

[13] V. Cortés, M. Dyckmanns, M. Jüngling and D. Lindemann, A class of cubic hypersurfaces and quaternionic Kähler manifolds of co-homogeneity one, arXiv:1701.07882 [INSPIRE].

[14] E. Cremmer et al., Vector Multiplets Coupled to $N=2$ Supergravity: SuperHiggs Effect, Flat Potentials and Geometric Structure, Nucl. Phys. B 250 (1985) 385 [INSPIRE]. 
[15] E. Cremmer and A. Van Proeyen, Classification of Kähler Manifolds in $N=2$ Vector Multiplet Supergravity Couplings, Class. Quant. Grav. 2 (1985) 445 [INSPIRE].

[16] B. de Wit, P.G. Lauwers, R. Philippe, S.Q. Su and A. Van Proeyen, Gauge and Matter Fields Coupled to $N=2$ Supergravity, Phys. Lett. B 134 (1984) 37 [inSPIRE].

[17] B. de Wit and A. Van Proeyen, Potentials and Symmetries of General Gauged N=2 Supergravity: Yang-Mills Models, Nucl. Phys. B 245 (1984) 89 [INSPIRE].

[18] B. de Wit and A. Van Proeyen, Special geometry, cubic polynomials and homogeneous quaternionic spaces, Commun. Math. Phys. 149 (1992) 307 [hep-th/9112027] [INSPIRE].

[19] S. Ferrara, G.W. Gibbons and R. Kallosh, Black holes and critical points in moduli space, Nucl. Phys. B 500 (1997) 75 [hep-th/9702103] [INSPIRE].

[20] S. Ferrara and M. Günaydin, Orbits of exceptional groups, duality and BPS states in string theory, Int. J. Mod. Phys. A 13 (1998) 2075 [hep-th/9708025] [INSPIRE].

[21] S. Ferrara, K. Hayakawa and A. Marrani, Lectures on Attractors and Black Holes, Fortsch. Phys. 56 (2008) 993 [arXiv:0805.2498] [InSPIRE].

[22] S. Ferrara and R. Kallosh, Supersymmetry and attractors, Phys. Rev. D 54 (1996) 1514 [hep-th/9602136] [INSPIRE].

[23] S. Ferrara and R. Kallosh, Universality of supersymmetric attractors, Phys. Rev. D 54 (1996) 1525 [hep-th/9603090] [INSPIRE].

[24] S. Ferrara, R. Kallosh and A. Strominger, $N=2$ extremal black holes, Phys. Rev. D 52 (1995) R5412 [hep-th/9508072] [INSPIRE].

[25] S. Ferrara and A. Strominger, $N=2$ space-time supersymmetry and Calabi-Yau moduli space, in proceedings of the Strings 89: International Superstring Workshop, College Station, TX, U.S.A., 13-18 March 1989, World Scientific, River Edge NJ U.S.A. (1990), pp. 245-252.

[26] B. van Geemen, A. Marrani and F. Russo, BPS Black Hole Entropy and Attractors in Very Special Geometry. Cubic Forms, Gradient Maps and their Inversion, arXiv:2009.10647 [INSPIRE].

[27] M. Günaydin, G. Sierra and P.K. Townsend, The Geometry of $N=2$ Maxwell-Einstein Supergravity and Jordan Algebras, Nucl. Phys. B 242 (1984) 244 [InSPIRE].

[28] S.W. Hawking, Gravitational radiation from colliding black holes, Phys. Rev. Lett. 26 (1971) 1344 [INSPIRE].

[29] R. Kallosh, Supersymmetric black holes, Phys. Lett. B 282 (1992) 80 [hep-th/9201029] [INSPIRE].

[30] C.I. Lazaroiu and C.S. Shahbazi, Generalized Einstein-Scalar-Maxwell theories and locally geometric U-folds, Rev. Math. Phys. 30 (2018) 1850012 [arXiv:1609.05872] [INSPIRE].

[31] S.D. Majumdar, A class of exact solutions of Einstein's field equations, Phys. Rev. 72 (1947) 390 [INSPIRE].

[32] A. Marrani, Non-linear symmetries in Maxwell-Einstein gravity: from Freudenthal duality to pre-homogeneous vector spaces, in Lie Theory and its Applications in Physics, Springer, Singapore (2020), pp. 253-264.

[33] K. McCrimmon, A taste of Jordan algebras, Springer-Verlag, New York NY U.S.A. (2004). 
[34] A. Papapetrou, A static solution of the equations of the gravitational field for an arbitrary charge distribution, Proc. Roy. Ir. Acad. A 51 (1947) 191.

[35] M.K. Prasad and C.M. Sommerfield, An Exact Classical Solution for the 't Hooft Monopole and the Julia-Zee Dyon, Phys. Rev. Lett. 35 (1975) 760 [inSPIRE].

[36] I. Robinson, A solution of the Maxwell-Einstein equations, Bull. Acad. Pol. Sci. Ser. Sci. Math. Astron. Phys. 7 (1959) 351.

[37] N. Seiberg and E. Witten, Electric-magnetic duality, monopole condensation, and confinement in $N=2$ supersymmetric Yang-Mills theory, Nucl. Phys. B 426 (1994) 19 [Erratum ibid. 430 (1994) 485] [hep-th/9407087] [INSPIRE].

[38] M. Shmakova, Calabi-Yau black holes, Phys. Rev. D 56 (1997) 540 [hep-th/9612076] [INSPIRE].

[39] A. Strominger, Special geometry, Commun. Math. Phys. 133 (1990) 163 [INSPIRE].

[40] È.B. Vinberg, The theory of convex homogeneous cones, Tr. Mosk. Mat. Obs. 12 (1963) 303.

[41] E. Witten and D.I. Olive, Supersymmetry Algebras That Include Topological Charges, Phys. Lett. B 78 (1978) 97 [INSPIRE]. 